\title{
Chromatic Polynomials for Families of Strip Graphs and their Asymptotic Limits
}

\author{
Martin Roček*, Robert Shrock ${ }^{\dagger}$, Shan-Ho Tsai ${ }^{\ddagger}$ \\ Institute for Theoretical Physics \\ State University of New York \\ Stony Brook, N. Y. 11794-3840
}

\begin{abstract}
We calculate the chromatic polynomials $P\left(\left(G_{s}\right)_{m}, q\right)$ and, from these, the asymptotic limiting functions $W\left(\left\{G_{s}\right\}, q\right)=\lim _{n \rightarrow \infty} P\left(G_{s}, q\right)^{1 / n}$ for families of $n$-vertex graphs $\left(G_{s}\right)_{m}$ comprised of $m$ repeated subgraphs $H$ adjoined to an initial graph $I$. These calculations of $W\left(\left\{G_{s}\right\}, q\right)$ for infinitely long strips of varying widths yield important insights into properties of $W(\Lambda, q)$ for two-dimensional lattices $\Lambda$. In turn, these results connect with statistical mechanics, since $W(\Lambda, q)$ is the ground state degeneracy of the $q$-state Potts model on the lattice $\Lambda$. For our calculations, we develop and use a generating function method, which enables us to determine both the chromatic polynomials of finite strip graphs and the resultant $W\left(\left\{G_{s}\right\}, q\right)$ function in the limit $n \rightarrow \infty$. From this, we obtain the exact continuous locus of points $\mathcal{B}$ where $W\left(\left\{G_{s}\right\}, q\right)$ is nonanalytic in the complex $q$ plane. This locus is shown to consist of arcs which do not separate the $q$ plane into disconnected regions. Zeros of chromatic polynomials are computed for finite strips and compared with the exact locus of singularities $\mathcal{B}$. We find that as the width of the infinitely long strips is increased, the arcs comprising $\mathcal{B}$ elongate and move toward each other, which enables one to understand the origin of closed regions that result for the (infinite) $2 \mathrm{D}$ lattice.
\end{abstract}

05.20.-y, 75.10.H, 02.10.Eb, 02.10.Rn

*email: rocek@insti.physics.sunysb.edu

${ }^{\dagger}$ corresponding author, email: shrock@insti.physics.sunysb.edu

${ }^{\ddagger}$ email: tsai@insti.physics.sunysb.edu 


\section{INTRODUCTION}

Nonzero ground state disorder and associated entropy, $S_{0} \neq 0$, is an important subject in statistical mechanics. One physical example is provided by ice, for which the residual molar entropy is $S_{0}=0.82 \pm$ $0.05 \mathrm{cal} /\left(\mathrm{K}\right.$-mole), i.e., $S_{0} / R=0.41 \pm 0.03$, where $R=N_{\text {Avog. }} k_{B}$ [1] 5 . Indeed, residual entropy at low temperatures has been observed in a number of substances, including nitrous oxide, NO, carbon monoxide, $\mathrm{CO}$, and $\mathrm{FClO}_{3}$ (a comprehensive review is given in Ref. [4]). In these examples, the entropy occurs without frustration. In magnetic systems, two examples are provided by the Ising antiferromagnet on the triangular and kagomé lattices [6.7]; here, the ground state entropy does involve frustration. One should also mention the recent experimental observation of compounds whose behavior can be modelled by quantum Heisenberg antiferromagnets on a kagomé lattice, including $\mathrm{SrCr}_{8-x} \mathrm{Ga}_{4+x} \mathrm{O}_{19},(\mathrm{SCGO}(\mathrm{x}))$ [ ] and deuteronium jarosite, $\left(\mathrm{D}_{3} \mathrm{O}\right) \mathrm{Fe}_{3}\left(\mathrm{SO}_{4}\right)_{2}(\mathrm{OD})_{6}[9]$. The quantum Heisenberg antiferromagnet has a disordered ground state with finite entropy and frustration on the kagomé lattice [10]. A particularly simple model exhibiting ground state entropy without the complication of frustration is the $q$-state Potts antiferromagnet (AF) [11, 12] on a lattice $\Lambda$, for $q \geq \chi(\Lambda)$, where $\chi(\Lambda)$ denotes the minimum number of colors necessary to color the vertices of the lattice such that no two adjacent vertices have the same color. As is already evident from the foregoing, this model also has a deep connection with graph theory since the zero-temperature partition function of the above-mentioned $q$-state Potts antiferromagnet on a lattice $\Lambda$ satisfies $Z(\Lambda, q, T=0)_{P A F}=P(\Lambda, q)$, where $P(G, q)$ is the chromatic polynomial expressing the number of ways of coloring the vertices of a graph $G$ I with $q$ colors such that no two adjacent vertices (connected by a bond of the graph) have the same color [13 18. Hence, the ground state entropy per site is given by $S_{0} / k_{B}=\ln W(\Lambda, q)$, where $W(\Lambda, q)$, the ground state degeneracy per site, is

$$
W(\Lambda, q)=\lim _{n \rightarrow \infty} P\left(\Lambda_{n}, q\right)^{1 / n}
$$

Here, $\Lambda_{n}$ denotes an $n$-vertex lattice of type $\Lambda$ (square, triangular, etc.), with appropriate (e.g., free) boundary conditions.

Since nontrivial exact solutions for this function are known in only a very few cases (square lattice for $q=3$ [19], triangular lattice [20], and kagomé lattice for $q=3$ [12]), it is important to exploit and extend general approximate methods that can be applied to all cases. Such methods include rigorous upper and lower bounds, large- $q$ series expansions, and Monte Carlo measurements. Recently, we studied the ground state entropy in antiferromagnetic Potts models on various lattices and obtained further results with these

\footnotetext{
${ }^{1}$ We recall the strict mathematical definition of a graph as a collection of vertices and bonds connecting (all or a subset of the) vertices, such that there are (i) no loops, i.e., bonds connecting a vertex to itself, (ii) no multiple bonds connecting a given pair of vertices, and (iii) the total number of vertices is finite. Thus, an infinite lattice is not a graph, but a limit thereof. A graph is termed empty if it has no bonds connecting the vertices.
} 
three methods 21 24]; see also Ref. [25]. As $P(G, q)$ is a polynomial, $q$ may be continued from integer to complex values, which we do here.

One can gain valuable insight into the values of the ground state degeneracy of the Potts antiferromagnet $W(\Lambda, q)$ for different 2D lattices $\Lambda$ and values of $q$ by studying this function on infinitely long strips of increasing widths. Accordingly, in this paper, we calculate $P\left(\left(G_{s}\right)_{m}, q\right)$ for a family of strip graphs $\left(G_{s}\right)_{m}$ which are comprised of $m$ repetitions of a subgraph unit $H$ attached to an initial subgraph $I$ on one end (which, by convention, we take to be the right end). Symbolically, we denote such a strip graph as

$$
\left(G_{s}\right)_{m}=\left(\prod_{\ell=1}^{m} H\right) I
$$

for $m \geq 1$, with $\left(G_{s}\right)_{0} \equiv I$. The initial subgraph $I$ may itself be equal to $H$. To carry out these calculations, we construct a generating function $\Gamma\left(G_{s}, q, x\right)$ that yields the chromatic polynomial $P\left(\left(G_{s}\right)_{m}, q\right)$ as the coefficient of the term $x^{m}$ in its Taylor series expansion about $x=0$, where $x$ is a formal auxiliary variable. We then use this generating function to compute the asymptotic limiting function for the infinitely long strip graph of type $G_{s}, W\left(\left\{G_{s}\right\}, q\right)$ defined in eq. (1.1), and to characterize its analytic properties in the complex $q$ plane. We do not connect the ends of the strip in the longitudinal direction, i.e., the strip has free boundary conditions in both the transverse and longitudinal directions. As in Refs. 221 24], we let $\mathcal{B}$ denote the continuous locus of points in the $q$ plane where $W\left(\left\{G_{s}\right\}, q\right)$ is nonanalytic. For the infinite-length limit of the strip graphs $G_{s}$ defined in eq. (1.2) this locus of points $\mathcal{B}$ consists of arcs which do not separate the complex $q$ plane into different regions. We calculate these for a number of different types of strips.

In general, in the limit as the number $n$ of vertices on an $n$-vertex graph of type $G$ goes to infinity, the locus $\mathcal{B}$ forms as a merging together of zeros of the chromatic polynomial $P(G, q)$ (called chromatic zeros of $G)$. It is thus of interest to calculate chromatic zeros for strip graphs $\left(G_{s}\right)_{m}$ of finite length and to compare these with the asymptotic limit represented by the curves making up $\mathcal{B}$. We perform this comparison for a number of types of strip graphs here.

Our results are useful for several reasons. First, they are a set of exact calculations of chromatic polynomials for a large variety of strips, of both finite and infinite length. Second, the generating function introduced here enables us to calculate, in an algorithmic manner, the chromatic polynomials for very long finite strips. To appreciate how valuable such a method is, we recall the well-known fact [16] that, in general, the calculation of the chromatic polynomial of an arbitrary graph is an NP-complete problem (NP = nonpolynomial), i.e., roughly speaking, it requires a time which grows exponentially as a function of the number of vertices in the graph. Third, the generating function provides an elegant way to obtain directly the infinite-length limiting function $W\left(\left\{G_{s}\right\}, q\right)$ for a given strip graph of type $G_{s}$. These $W(\{G\}, q)$ functions show a rich and fascinating analytic structure in the complex $q$ plane. Fourth, as noted before, by studying this structure as a function of the width of the strips, one gains insight into how the limit of the infinite 2D lattice is approached (with free boundary conditions).

We define 


$$
W(\{G\}, q) \equiv W\left(\{G\}, q_{s}\right)_{D_{q n}} \equiv \lim _{q \rightarrow q_{s}} \lim _{n \rightarrow \infty} P(G, q)^{1 / n}
$$

and recall from Ref. [21] that at certain special points $q_{s}$, the following limits do not commute:

$$
\lim _{n \rightarrow \infty} \lim _{q \rightarrow q_{s}} P(G, q)^{1 / n} \neq \lim _{q \rightarrow q_{s}} \lim _{n \rightarrow \infty} P(G, q)^{1 / n}
$$

Our present definition (1.3) is the same as in Ref. [21] and has the advantage of maintaining the analyticity of $W(\{G\}, q)$ at the special points $q_{s}$.

In Ref. [21] two of us had also discussed a second subtlety in the definition of $W(\{G\}, q)$ : for certain ranges of real $q, P(G, q)$ can be negative, and, of course, when $q$ is complex, so is $P(G, q)$ in general. In these cases it may not be obvious, a priori, which of the $n$ roots

$$
P(G, q)^{1 / n}=\left\{|P(G, q)|^{1 / n} e^{2 \pi i r / n}\right\}, \quad r=0,1, \ldots, n-1
$$

to choose in eq. (1.1). Consider the function $W(\{G\}, q)$ defined via eq. (1.1) starting with $q$ on the positive real axis where $P(G, q)>0$, and consider the maximal region in the complex $q$ plane that can be reached by analytic continuation of this function. We denote this region as $R_{1}$. Clearly, the phase choice in (1.5) for $q \in R_{1}$ is that given by $r=0$, namely $P(G, q)^{1 / n}=|P(G, q)|^{1 / n}$. For the various exactly solved cases in Ref. 21] concerned with families of graphs $\{G\}$ for which the areas of analyticity of $W(\{G\}, q)$ include other regions not analytically connected to $R_{1}$, there is not, in general, any canonical choice of phase in (1..5). However, for the strip graphs studied here, the curves $\mathcal{B}$ where $W\left(\left\{G_{s}\right\}, q\right)$ is nonanalytic do not separate the $q$ plane into different regions, i.e., the entire $q$ plane, except for the locus $\mathcal{B}$, is in $R_{1}$, so this second point having to do with phase choice in (1.5) is easily dealt with, as discussed below.

This paper is organized as follows. In Section II we introduce the generating function. In Section III we calculate the generating functions for a number of interesting types of strip graphs $G_{s}$. Section IV is devoted to a study of the analytic properties of the resultant $W\left(\left\{G_{s}\right\}, q\right)$ functions. Some final remarks are given in the conclusions. Besides the mathematical references [13 18] and the previous Refs. [21 24] by two of us, some related work is in Refs. [19,26,27,20,28]. We shall report further results in Ref. [29].

\section{CALCULATION OF $P\left(G_{s}, q\right)$ VIA A GENERATING FUNCTION}

\section{A. Generalities}

It is possible to calculate the chromatic polynomials for a wide variety of strip graphs $\left(G_{s}\right)_{m}$ of the type defined in eq. (1.2) by computing a generating function. This also enables us immediately to calculate the resultant asymptotic limiting function $W\left(\left\{G_{s}\right\}, q\right)$. Before proceeding, we discuss some preliminaries. We denote the number of vertices in a graph $G$ as $n(G)$. Consider two successive repeating subgraph units $H$ and denote their intersection as the graph

$$
L_{H}=H_{r+1} \cap H_{r}
$$


Let the number of vertices in the repeating subgraph unit $H$, the intersection graph $L_{H}$, and the initial graph $I$ be $n(H), n\left(L_{H}\right)$, and $n(I)$, respectively. Then the total number of vertices $n$ in the strip graph $\left(G_{s}\right)_{m}$ is

$$
n\left(\left(G_{s}\right)_{m}\right)=c_{s, 1} m+c_{s, 0}
$$

where

$$
c_{s, 0}=n(I)
$$

and

$$
c_{s, 1}=n(H)-n\left(L_{H}\right)
$$

The values of these coefficients are listed in Tables I and II for the various strip graphs that we consider.

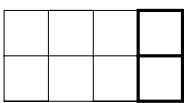

(a)

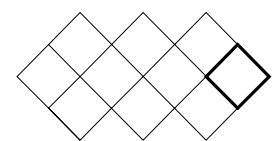

(c)

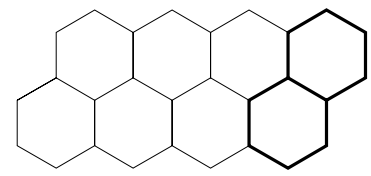

(e)

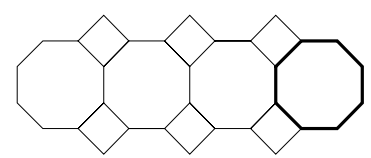

(g)

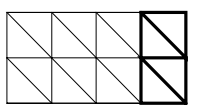

(b)

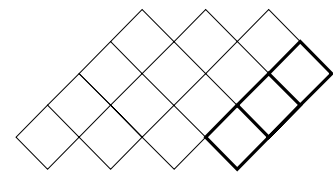

(d)

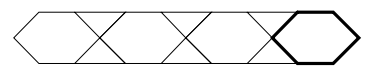

(f)

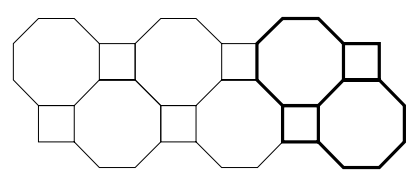

(h) 
FIG. 1. Illustrations of strip graphs $\left(G_{s\left(L_{y}\right)}\right)_{m}$ of type $s$. Subscripts $L_{y}$ and $m$ refer, respectively, to the width and the number of repeated subgraph units $H$ beyond the initial subgraph $I$ (where $I$ may be identical to $H$ ). For (a)-(g), $m=3$, whereas $m=2$ for $(\mathrm{h})$. For ease of identification, the initial subgraph $I$ is drawn with heavier lines. See text for further discussion of each type of strip graph. (a) $\left(G_{s q(3)}\right)_{3}$, an $L_{x} \times L_{y}=5 \times 3$ strip of the square lattice; (b) $\left(G_{\operatorname{tri}(3)}\right)_{3}$, an $L_{x} \times L_{y}=5 \times 3$ strip of the triangular lattice (represented as a square lattice with additional oblique bonds); (c) $\left(G_{s q_{d}}(3)\right)_{3}$, a strip of a square lattice along the diagonal direction, with the repeating subgraph $H$ comprised of the upper, lower, and left members of a $3 \times 3$ diagonally oriented box; (d) $\left(G_{s q_{d}(4)}\right)_{3}$, as in (c), but with a repeating unit $H$ comprised of a diagonally oriented strip of 4 squares; (e) $\left(G_{h c(3)}\right)_{3}$, a strip of the honeycomb lattice; (f) $\left(G_{k a g(2)}\right)_{3}$, a strip of the kagomé lattice; $(\mathrm{g})\left(G_{488(2)}\right)_{3}$, a strip of the $\left(4 \cdot 8^{2}\right)$ lattice; $(\mathrm{h})\left(G_{488(3)}\right)_{2}$, a wider strip of the $\left(4 \cdot 8^{2}\right)$ lattice along a direction rotated $45^{\circ}$ relative to that in $(\mathrm{g})$.

For example, consider strips of the square, triangular, and honeycomb lattices, as shown in Figs. 11(a,b,e). We represent the strip of the triangular lattice as a strip of squares with oblique bonds added, as indicated in Fig. 1(b). For our present discussion we represent the honeycomb lattice of Fig. 1(e) as a brick lattice. Denote the length as $L_{x}$ vertices in the horizontal (longitudinal) direction, and the width as $L_{y}$ vertices in the vertical (transverse) direction. This corresponds to a width of $w=L_{y}-1$ layers of squares or hexagons and $2 w$ triangles, for the respective lattices. The strips of the square and triangular lattices shown in Fig. 1 1 (a,b) have $L_{x}=5$ and $L_{y}=3$. These strips are thus $L_{x} \times L_{y}$ lattices with free boundary conditions in both $x$ and $y$ directions. The strip of the honeycomb lattice of Fig. If(e), when represented in brick form, has $L_{x}=9$ for the top and bottom edges and $L_{x}=10$ for the interior layer, with $L_{y}=3$. Later we will study the limit in which $L_{x} \rightarrow \infty$ with $L_{y}$ fixed, i.e., strips of size $\infty \times L_{y}$. The square strip has, as a repeating subgraph unit $H$, a vertical stack of $w$ squares. Hence, starting at one end, which will be taken by convention to be on the right and to consist of an initial subgraph $I$ which is identical to $H$, the elongation of the strip proceeds by the addition of successive vertical stacks on the growing (left) end. For the honeycomb and triangular lattices, respectively, $H$ is an oblique stack of $w$ hexagons and a vertical stack of $2 w$ triangles, and the initial subgraph $I=H$. For the strips of the square lattice (along the row direction) and of the triangular lattice, the relation between $L_{x}$ and $m$ is thus $L_{x}=m+2$, and

$$
n(H)=2 n\left(L_{H}\right)=2 L_{y} \quad \text { for } \quad G_{s q\left(L_{y}\right)} \quad \text { or } \quad G_{t\left(L_{y}\right)}
$$

while for the honeycomb lattice,

$$
n(H)=2\left(2 L_{y}-1\right), \quad n\left(L_{H}\right)=2\left(L_{y}-1\right) \quad \text { for } \quad G_{h c\left(L_{y}\right)}
$$

The number $n(I)$ depends on one's convention for the initial subgraph; in these three types of strips, since we take $I=H$, we have $n(I)=n(H)$ for each. The total number of vertices is $n\left(\left(G_{s\left(L_{y}\right)}\right)_{m}\right)=L_{x} L_{y}=(m+2) L_{y}$ for the square and triangular lattices, so

$$
c_{s\left(L_{y}\right), 1}=L_{y}, \quad c_{s\left(L_{y}\right), 0}=2 L_{y} \quad \text { for } \quad s=s q, t
$$

while for the honeycomb (brick) lattice, $n\left(\left(G_{h c\left(L_{y}\right)}\right)_{m}\right)=2\left[(m+2) L_{y}-1\right]$, so

$$
c_{h c\left(L_{y}\right), 1}=2 L_{y}, \quad c_{h c\left(L_{y}\right), 0}=2\left(2 L_{y}-1\right)
$$

It is straightforward to obtain the analogous formulas for other types of strip graphs $G_{s}$; we list the results for $c_{s, 1}$ and $c_{s, 0}$ in Tables I and II. 


\section{B. Main Theorem and Proof}

The generating function for a strip of type $G_{s}$, denoted generically as $\Gamma\left(G_{s}, q, x\right)$, yields the chromatic polynomials via the expansion

$$
\Gamma\left(G_{s}, q, x\right)=\sum_{m=0}^{\infty} P\left(\left(G_{s}\right)_{m}, q\right) x^{m}
$$

where $x$ is a symbolic variable and $P\left(\left(G_{s}\right)_{m}, q\right)$ is the chromatic polynomial for the coloring, with $q$ colors, of the strip $\left(G_{s}\right)_{m}$ with $m$ repeated subgraph units $H$ beyond the initial $(m=0)$ one, $I$. We find that the generating function $\Gamma\left(G_{s}, q, x\right)$ is a rational function of the form

$$
\Gamma\left(G_{s}, q, x\right)=\frac{\mathcal{N}\left(G_{s}, q, x\right)}{\mathcal{D}\left(G_{s}, q, x\right)}
$$

with

$$
\mathcal{N}\left(G_{s}, q, x\right)=\sum_{j=0}^{j_{\max }} a_{s, j}(q) x^{j}
$$

and

$$
\mathcal{D}\left(G_{s}, q, x\right)=1+\sum_{k=1}^{k_{\max }} b_{s, k}(q) x^{k}
$$

where the $a_{s, i}$ and $b_{s, i}$ are polynomials in $q$ that depend on the type and width of the strip graph $G_{s}$. The polynomials $a_{s, i}$ also depend on the subgraph forming the right-hand end of the strip (discussed further below), while the $b_{s, i}$ are independent of this subgraph. The respective degrees $j_{\max }$ and $k_{\max }$ of the numerator and denominator polynomials $\mathcal{N}\left(G_{s}, q, x\right)$ and $\mathcal{D}\left(G_{s}, q, x\right)$, as functions of $x$, depend on the type and width of the strip $G_{s}$.

We can state these results formally:

\section{Theorem 1.}

Let a strip graph of type $G_{s}$ be constructed by $m$ successive additions of a repeating graphical subunit $H$ to an initial subgraph $I$ as in eq. (1.2). Then the chromatic polynomial for this strip graph can be expressed via the generating function (2.9) given by (2.10)-(2.12).

Proof

We use the deletion-contraction theorem, which states that the chromatic polynomial for a graph $G$ containing two adjacent vertices $v$ and $v^{\prime}$ connected by a bond (edge) is equal to the chromatic polynomial for a graph in which this bond is removed minus the chromatic polynomial for the graph in which these 
vertices are identified2. Applying the deletion-contraction theorem to the initial subgraph $I$, we obtain a finite set of linear equations, with $m$-independent coefficients, for $P\left(\left(G_{s}\right)_{m}, q\right), P\left(\left(G_{s}\right)_{m-1}, q\right), P\left(\left(G_{s, e}\right)_{m}, q\right)$, and possibly $P\left(\left(G_{s, e}\right)_{m-1}, q\right)$, where $G_{s, e}$ denotes a strip graph of type $s$ but with a different end subgraph (e) at the starting point on the right. In general, the application of the deletion-contraction theorem yields several $G_{s, e}$ 's with different end subgraphs $e$. The set of equations is linear because the deletion-contraction theorem is a linear equation among chromatic polynomials. The set of linear equations is finite because the repeating subgraph unit $H$ is a finite graph. Next, assuming the form (2.9) for each type of strip and substituting into these equations, we obtain a resultant set of linear equations for the generating function of the original strip, $\Gamma\left(G_{s}, q, x\right)$, and the set of generating functions for the strips with different ends produced by the action of the deletion-contraction theorem, $\Gamma\left(G_{s, e}, q\right)$, which we solve. This completes the proof.

We illustrate the method of proof by an explicit example. We first note that a graph in which each adjacent pair of repeating subgraph units $H$ intersect each other in a complete graph $L_{H}=K_{\ell}$, has a very simple chromatic polynomial. Since the intersection of $H$ with $L_{H}$ is just $L_{H}$, the intersection theorem theory implies that the chromatic polynomial $P(H, q)$ factorizes according to

$$
P(H, q)=P\left(K_{\ell}, q\right) P_{r}(H, q)
$$

where

$$
P\left(K_{\ell}, q\right)=\prod_{t=0}^{\ell-1}(q-t)
$$

which defines the polynomial $P_{r}(H, q)$. For a chain graph $\left(G_{s ; K_{\ell}}\right)_{m}$ consisting of $m$ repeated subgraphs $H$ with an initial subgraph $I=H$, the chromatic polynomial is easily computed by the interative use of the intersection theorem:

$$
P\left(\left(G_{s ; K_{\ell}}\right)_{m}, q\right)=\frac{P(H, q)^{m+1}}{P\left(K_{\ell}, q\right)^{m}}=\left(\prod_{t=0}^{\ell-1}(q-t)\right) P_{r}(H, q)^{m+1}
$$

The generating function is

\footnotetext{
2 The deletion-contraction theorem is an expression of the elementary fact the chromatic polynomial of $G$ enumerates the coloring of the graph such that the colors of each pair of adjacent vertices are different. In particular, then, the number of colorings in which the colors on the pair $v$ and $v^{\prime}$ are different is equal to the number of colorings in which the colors assigned to $v$ and $v^{\prime}$ are allowed to vary freely (subject to the constraints from their other bonds) minus the number in which the colors assigned to $v$ and $v^{\prime}$ are the same. Reading this equation in the opposite direction gives the addition-contraction theorem of graph theory, which we shall use later. See, e.g., Refs. [16]- [18] for further discussion of the mathematical background.

${ }^{3}$ The complete graph $K_{\ell}$ is defined as the graph consisting of $\ell$ vertices, each of which is connected to all of the others with bonds.

${ }^{4}$ The intersection theorem states that if two graphs $G$ and $G^{\prime}$ intersect in a complete graph $K_{\ell}$ for some $\ell$, then the chromatic polynomial of the union of these graphs is given by $P\left(G \cup G^{\prime}, q\right)=P(G, q) P\left(G^{\prime}, q\right) / P\left(K_{\ell}, q\right)$.
} 


$$
\Gamma\left(G_{s ; K_{\ell}}, q, x\right)=\frac{P(H, q)}{1-P_{r}(H, q) x}
$$

For example, for a strip of $m p$-sided polygons ( $p$-gons) with each adjacent pair of $p$-gons intersecting on a mutual edge $\left(K_{2}\right)$, the chromatic polynomial is

$$
P\left(\left(G_{p-g o n ; K_{2}}\right)_{m}, q\right)=q(q-1) D_{p}(q)^{m}
$$

where

$$
D_{p}(q)=\frac{P\left(C_{p}, q\right)}{q(q-1)}=\sum_{s=0}^{p-2}(-1)^{s}\left(\begin{array}{c}
p-1 \\
s
\end{array}\right) q^{p-2-s}
$$

and the chromatic polynomial for a circuit with $p$ vertices is

$$
P\left(C_{p}, q\right)=(q-1)^{p}+(-1)^{p}(q-1)
$$

so that $D_{3}(q)=q-2$ and $D_{4}(q)=q^{2}-3 q+3$. (In the following, for brevity, we shall generally write out the expression for $D_{3}(q)$ but not for $D_{k}(q)$ for higher values of $k$.) Since the chromatic polynomial can easily be calculated for this type of strip without the use of our more powerful methods, and since, as will be discussed below, the points in the complex $q$ plane where the resultant $W\left(\left\{G_{s}\right\}, q\right)$ function is nonanalytic are at most isolated branch points, so that $\mathcal{B}=\emptyset$, i.e., the null set, we concentrate on more complicated types of strip graphs (e.g. of greater width) where the factorization in eq. (2.15) does not occur. 


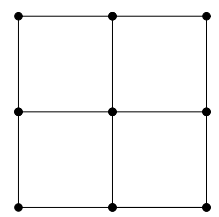

(a)

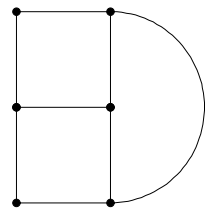

(d)

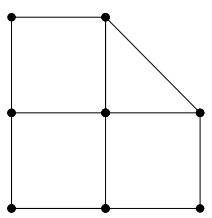

(b)

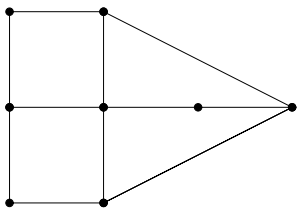

(e)

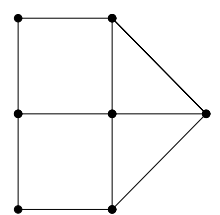

(c)

FIG. 2. Graphs for square strips of width $L_{y}=3$ of type (a) $G_{s q, m=1}$ (b) $G_{s q, e=s t, m=1}$, (c) $G_{s q, e=t t, m=1}$, (d) $G_{s q, e=t, m=1}$, and (e) $G_{s q, e=t t v, m=1}$. See text for discussion.

Let us, then, illustrate our method of proof by considering a strip graph that consists of squares, $G_{s q}$. The method consists of using the deletion-contraction theorem on the end of the original strip graph, which generates also related strip graphs with different initial subgraphs; one then uses the deletion-contraction on these, thereby generating further types of strip graphs, and so forth until no further additional types of strip graphs are generated. (Of course, all of these additional types of strip graphs also have free boundary conditions.) In this manner, one obtains a set of linear equations for the generating functions of all of these types of strip graphs, which is then solved. A general strip of this type has size $L_{x} \times L_{y}$ vertices, i.e., $(m+1) \times w$ squares, where $m=L_{x}-2$ and $w=L_{y}-1$, as given above. Both the strip with $L_{y}=1$ (the line) and $L_{y}=2$ have chromatic polynomials that factorize, so we consider the next simplest case of width $L_{y}=3$ (see Fig. 2(a)). To simplify the notation as much as possible, we shall use the symbols 
$P_{s\left(L_{y}, e\right), m} \equiv P\left(\left(G_{s\left(L_{y}\right),(e)}\right)_{m}, q\right)$ and shall put $G_{s q\left(L_{y}=3\right)} \equiv G_{s q(3)} \equiv G_{s s}$. A first application of the deletioncontraction theorem on one of the vertical bonds (say, the upper one) at the right end of a length- $m$ strip yields the equation

$$
P_{s s, m}=(q-1) D_{4}(q) P_{s s, m-1}-P_{s t, m}
$$

for $m \geq 1$, where $P_{s t, m}$ refers to the chromatic polynomial for the graph $G_{s q, e=s t, m}$ with right-hand end consisting of a square and triangle (whence the subscript st) shown in Fig. 2(b). Given our convention of taking $G_{s q, m=0}$ to be the graph with two squares on top of each other and $G_{s q, e=s t, m=0}$ to be a graph with a triangle adjoined to a square, it follows that

$$
P_{s s, 0} \equiv P\left(\left(G_{s q(2)}\right)_{0}, q\right)=q(q-1) D_{4}(q)^{2}
$$

and

$$
P_{s t, 0}=q(q-1)(q-2) D_{4}(q)
$$

Equation 2.20) thus relates the chromatic polynomial of the square strip of length $m$ and width 2 to the chromatic polynomial of the same type of strip of length $m-1$ and to the chromatic polynomial of this type of square strip with a different type of endgraph, as shown in Fig. 2(b). We next apply the deletion-contraction theorem to this latter type of strip, specifically to the remaining vertical end bond on the right in Fig. 2(b), to obtain the relation

$$
P_{s t, m}=(q-1)(q-2) P_{s s, m-1}-P_{t t, m}
$$

for $m \geq 1$, and $P_{t t, m}$ is the chromatic polynomial for the square strip graph $G_{s q, e=t t, m}$ with a right-hand end consisting of two triangles, as shown in Fig. 2( (c). Here we take $G_{s q, e=t t, m=0}$ to be the two triangles adjoined along their horizontal edge, so that

$$
P_{t t, 0}=q(q-1)(q-2)^{2}
$$

Proceeding by applying the theorem to one of the hypotenuses of the triangles in $G_{s q, e=t t, m}$, we derive

$$
P_{t t, m}=(q-2) P_{s s, m-1}-P_{t, m}
$$

for $m \geq 1$, where the graph $G_{s q, e=t, m=1}$ is shown in Fig. 2 (d), so that the $m=0$ graph of this type is a single triangle, with $P_{t, 0}=P\left(K_{3}, q\right)=q(q-1)(q-2)$. The next deletion, on the round bond on the right in Fig. 2(d), yields

$$
P_{t, m}=P_{s s, m-1}-P_{t t v, m-1}
$$

for $m \geq 1$, where the graph $G_{s q, e=t t v, m=1}$ is shown in Fig. 2 (e), and the $m=0$ graph of this type is taken to be the 5 -vertex one at the right, with 


$$
P_{t t v, 0}=q(q-1)\left(q^{3}-5 q^{2}+10 q-7\right)
$$

Deleting one of the oblique bonds at the right end in Fig. 2(e) gives the relation

$$
P_{t t v, m}=D_{4}(q) P_{s s, m-1}-(q-2) P_{t, m}
$$

for $m \geq 1$. Evidently, these linear relations have now closed, i.e., no new types of strip graphs are introduced beyond the $s s, s t, t t, t, t t v$ that we have. Next, we substitute the assumed form (2.9) into these equations. Note that we do not have to specify $j_{\max }$ or $k_{\max }$; these are determined automatically by our solution of the equations. This substitution yields five linear equations for the five generating functions for the different types of strips: the original one, $\Gamma_{s s}$, together with the other four that have been generated by the operations of the deletion-contraction theorem. This set of equations can be written as the linear transformation

$$
T v_{\Gamma}=v_{0}
$$

where the vectors $v_{\Gamma}$ and $v_{0}$ are defined as

$$
\begin{gathered}
v_{\Gamma}=\left(\begin{array}{c}
\Gamma_{s s} \\
\Gamma_{s t} \\
\Gamma_{t t} \\
\Gamma_{t} \\
\Gamma_{t t v}
\end{array}\right) \\
v_{0}=\left(\begin{array}{c}
P_{s s, 0}+P_{s t, 0} \\
P_{s t, 0}+P_{t t, 0} \\
P_{t t, 0}+P_{t, 0} \\
P_{t, 0} \\
P_{t t v, 0}+(q-2) P_{t, 0}
\end{array}\right)
\end{gathered}
$$

and, with this ordering of the basis, the matrix $T$ is given by

$$
T=\left(\begin{array}{ccccc}
1-(q-1) D_{4}(q) x & 1 & 0 & 0 & 0 \\
-(q-1)(q-2) x & 1 & 1 & 0 & 0 \\
-(q-2) x & 0 & 1 & 1 & 0 \\
-x & 0 & 0 & 1 & x \\
-D_{4}(q) x & 0 & 0 & (q-2) & 1
\end{array}\right)
$$

The solution to eq. (2.29), $v_{\Gamma}=T^{-1} v_{0}$, yields the desired generating function $\Gamma_{s s}$, together with generating functions for the four other square strips of width 2 and different subgraphs on the right-hand ends. In particular, all of the five generating functions in $v_{\Gamma}$ have the same denominator $\mathcal{D}$ given as the determinant

$$
\mathcal{D}=\operatorname{det}(T)
$$

which is a general relation; in the present case,

$$
\mathcal{D}=1+\sum_{k=1}^{2} b_{s q(3), k} x^{k}
$$


where

$$
b_{s q(3), 1}=-(q-2)\left(q^{2}-3 q+5\right)
$$

and

$$
b_{s q(3), 2}=(q-1)\left(q^{3}-6 q^{2}+13 q-11\right)
$$

Note the general property that

$$
\operatorname{det}(T(x=0))=1
$$

whence

$$
\mathcal{D}(x=0)=1
$$

which was already incorporated into our form (2.10)-(2.12) with $b_{0}=1$. For all of the five strips, we get $j_{\max }=2$, i.e., the respective generating functions have the form

$$
\Gamma\left(G_{s\left(L_{y}\right) ;(e)}, q, x\right)=\frac{a_{s\left(L_{y}\right) ;(e), 0}+a_{s\left(L_{y}\right) ;(e), 1} x}{1+b_{s\left(L_{y}\right), 1} x+b_{s\left(L_{y}\right), 2} x^{2}}
$$

where, as above, the subscript (e) denotes the initial (end) subgraph and is implicit for the original strip, since its definition specifies the initial subgraph $I$. For brevity of notation, we shall sometimes also suppress the $\left(L_{y}\right)$ subscript, understanding it to be part of the type $s$. For the original square strip itself, the terms in the numerator polynomial $\mathcal{N}$ are

$$
\begin{gathered}
a_{s q(3), 0}=q(q-1) D_{4}(q)^{2} \\
a_{s q(3), 1}=-q(q-1)^{3}\left(q^{3}-6 q^{2}+13 q-11\right)
\end{gathered}
$$

From the results above, it is straightforward to obtain the numerators for the other four generating functions of the strips with different subgraphs at the right. For example, the numerator for $\Gamma_{t t} \equiv$ $\Gamma\left(G_{s q\left(L_{y}=3, e=t t\right)}, q, x\right)$ has

$$
\begin{gathered}
a_{s q\left(L_{y}=3, e=t t\right), 0}=P_{t t, 0}=q(q-1)(q-2)^{2} \\
a_{s q\left(L_{y}=3, e=t t\right), 1}=-q(q-1)^{2}(q-2)(q-3)
\end{gathered}
$$

In Appendix 1 we present two alternate ways of calculating the generating function $\Gamma\left(G_{s}, q, x\right)$ that are complementary to the method given above. 


\section{Some Basic Properties of Generating Functions}

In this subsection we prove some basic properties of generating functions using elementary properties of chromatic polynomials of arbitrary graphs. First, we observe that the zeroth order term in eq. (2.9) is just the chromatic polynomial of the initial subgraph $I$ :

$$
\Gamma\left(G_{s}, q, 0\right)=\mathcal{N}\left(G_{s}, q, 0\right)=a_{s, 0}=P(I, q)
$$

Next, we have

Theorem 2

$$
\Gamma\left(G_{s}, q, x\right)=0 \quad \text { for } \quad q=0,1, \ldots \ell-1 \quad \text { if } \quad G_{s} \supseteq K_{\ell}
$$

whence

$$
\begin{aligned}
& \mathcal{N}\left(G_{s}, q, x\right)=0 \quad \text { for } \quad q=0,1, \ldots \ell-1 \quad \text { if } \quad G_{s} \supseteq K_{\ell} \\
& a_{s, j}(q)=0 \quad \text { for } \quad q=0,1, \ldots \ell-1 \quad \forall j \quad \text { if } \quad G_{s} \supseteq K_{\ell}
\end{aligned}
$$

where $K_{\ell}$ denotes the complete graph with $\ell$ vertices.

Proof

One requires at least $\ell$ colors to color a complete graph $K_{\ell}$ subject to the condition that no adjacent vertices have the same color (all vertices are adjacent on a complete graph), i.e., $P\left(K_{\ell}, q\right)=0$ for $q=0,1, \ldots \ell-1$. It follows that if $G$ is an arbitrary graph that contains at least one complete graph $K_{\ell}$, then $P(G, q)=0$ for $q=0,1, \ldots, \ell-1$. In particular, this applies for the special case $G=G_{s}$.

Since $K_{1}$ is just a vertex, and $K_{2}$ is just a bond, and since all $G_{s}$ graphs have bonds (indeed have no disjoint vertices), a general consequence of Theorem 2 is that

$$
\Gamma\left(G_{s}, q, x\right)=0 \quad \text { for } \quad q=0,1
$$

whence

$$
\begin{gathered}
\mathcal{N}\left(G_{s}, q, x\right)=0 \quad \text { for } \quad q=0,1 \\
a_{s, j}(q)=0, \quad \text { for } \quad q=0,1 \quad \forall j
\end{gathered}
$$

The case $\ell=3$ in eqs. 2.45)-(2.47), where the subgraph is a triangle, $K_{3}$, applies to our strip graphs of the triangular, kagomé, and $\left(3 \cdot 12^{2}\right)$ lattices. 
Another general result follows from the fact that an elementary upper bound on a chromatic polynomial is $P(G, q) \leq q^{n}$ (for integer $q$ ) since the right-hand side is the number of ways that one can color the $n$-vertex graph $G$ without any constraint. Indeed, this is what motivates the use of the $1 / n$ 'th power in the definition of $W(\{G\}, q)$ in eq. (1.2). Since $P(G, q)$ is a polynomial, this bound also applies for $q$ generalized from integer to real and complex values. It follows that $W(\{G\}, q)$ is bounded above by $W(\{G\}, q) \leq q$ for real $q$, and, more generally, $|W(\{G\}, q)| \leq|q|$ for complex $q$. It is thus convenient to define, as we have in our earlier work [21 23], the reduced function

$$
W_{r}(\{G\}, q)=q^{-1} W(\{G\}, q)
$$

which has a finite limit as $|q| \rightarrow \infty$. In the case of regular lattices $\{G\}=\Lambda$, in the context of large- $q$ Taylor expansions of reduced $W$ functions [30,21,22] it is also useful to define a related reduced function

$$
\bar{W}(\Lambda, q)=\frac{W(\Lambda, q)}{q\left(1-q^{-1}\right)^{\zeta / 2}}
$$

where $\zeta$ is the coordination number of the lattice (in graph theory terminology, the degree $\Delta$ of a vertex on the lattice). The large- $q$ series expansion in terms of the variable

$$
y=\frac{1}{q-1}
$$

is then

$$
\bar{W}(\Lambda, y)=1+\sum_{n=1}^{\infty} w_{n} y^{n}
$$

(This assumes that $\bar{W}(\Lambda, y)$ is analytic at $y=1 / q=0$; for a recent discussion of this, see Refs. [21,23].) We shall discuss these large- $q$ series below in connection with our exact solutions for $W\left(\left\{G_{s}\right\}, q\right)$ for various strip graphs.

For strip graphs $\left(G_{s, k p}\right)_{m}$ where $G_{s}$ is $k$-partite $(k p)$, i.e., can be uniquely colored with $k$ colors such that no two adjacent vertices have the same color, the generating function satisfies

$$
\Gamma\left(G_{s, k p}, q=k, x\right)=\frac{k !}{1-x}
$$

reflecting the fact that

$$
P\left(\left(G_{s, k p}\right)_{m}, k\right)=k !
$$

\section{RESULTS FOR EXPLICIT GENERATING FUNCTIONS}

In this section we give results of our calculation of explicit generating functions for various types of strips. Since some of these, in particular, those associated with heteropolygonal Archimedean lattices, involve 
repeating subgraph units $H$ more complicated than just single polygons, it is useful to give formulas for the number of vertices as a function of the length of the strip, as measured by the number, $m$, of repeating subunits added to the initial subgraph $I$ forming the end of the strip. We do this in Tables I and II. These tables also contain information on the degrees of the polynomials $a_{s, j}$ and $b_{s, k}$ in the numerator and denominator of the generating functions for various types of strip graphs. As was evident in our proof of Theorem 1 and will be discussed further in section IV (see Corollaries 3.1 and 3.2), the choice of the initial subgraph $I$ affects only $\mathcal{N}$ and not $\mathcal{D}$ in the generating function and consequently does not affect the asymptotic limiting function $W\left(\left\{G_{s}\right\}, q\right)$.

TABLE I. Properties of generating functions for strip graphs $\left(G_{s}\right)_{m}$ as defined in eq. (1.2) of width $L_{y}$, comprised of $m$ repetitions of a subgraph $H$, with an initial subgraph $I$, as discussed in the text, and having $j_{\max }=1$ and $k_{\max }=2$. See the text for the definition of the diagonal width $d$. The degrees of $a_{s\left(L_{y}\right), j}$ and $b_{s\left(L_{y}\right), k}$, as polynomials in $q$, are denoted $\operatorname{deg}\left(a_{s\left(L_{y}\right), j}\right)$ and $\operatorname{deg}\left(b_{s\left(L_{y}\right), k}\right)$, and the subscript $s\left(L_{y}\right)$ is omitted to save space. $R_{s}$ is the discriminant defined in eq. 4.14 $N_{\text {b.p. }}$ is the number of branch point singularities in the degeneracy equation 4.16 ). $N_{\text {arcs }}$ and $N_{\text {s.c.arcs }}$ denote the number of arcs and self-conjugate arcs, respectively, in the continuous locus of points $\mathcal{B}$ where $W\left(\left\{G_{s}\right\}, q\right)$ is nonanalytic.

\begin{tabular}{|c|c|c|c|c|c|c|c|c|c|}
\hline$G_{s}$ & $c_{s, 1}$ & $c_{s, 0}=\operatorname{deg}\left(a_{0}\right)$ & $\operatorname{deg}\left(a_{1}\right)$ & $\operatorname{deg}\left(b_{1}\right)$ & $\operatorname{deg}\left(b_{2}\right)$ & $\operatorname{deg}\left(R_{s}\right)$ & $N_{\text {b.p. }}$. & $N_{\text {arcs }}$ & $N_{\text {s.c.arcs }}$ \\
\hline $\mathrm{sq}, L_{y}=3$ & 3 & 6 & 7 & 3 & 4 & 6 & 6 & 3 & 1 \\
\hline $\mathrm{sq}_{d}, d=3$ & 5 & 4 & 5 & 5 & 6 & 10 & 10 & 5 & 1 \\
\hline $\operatorname{tri}, L_{y}=3$ & 3 & 6 & 7 & 3 & 4 & 6 & 6 & 3 & 1 \\
\hline$(3 \cdot 6 \cdot 3 \cdot 6), L_{y}=2$ & 5 & 6 & 7 & 5 & 6 & 10 & 8 & 4 & 0 \\
\hline$\left(3 \cdot 12^{2}\right), L_{y}=2$ & 10 & 12 & 14 & 10 & 12 & 20 & 18 & 9 & 1 \\
\hline$\left(4 \cdot 8^{2}\right), L_{y}=2$ & 8 & 8 & 10 & 8 & 10 & 16 & 16 & 8 & 0 \\
\hline
\end{tabular}

TABLE II. Properties of generating functions for strip graphs $\left(G_{s}\right)_{m}$ with $j_{\max }>1, k_{\max }>2$. Notation is the same as in Table 1.

\begin{tabular}{|c|c|c|c|c|c|c|c|c|c|c|c|}
\hline$s$ & $j_{\max }$ & $k_{\max }$ & $c_{s, 1}$ & $c_{s, 0}=\operatorname{deg}\left(a_{0}\right)$ & $\operatorname{deg}\left(a_{1}\right)$ & $\operatorname{deg}\left(a_{2}\right)$ & $\operatorname{deg}\left(a_{3}\right)$ & $\operatorname{deg}\left(b_{1}\right)$ & $\operatorname{deg}\left(b_{2}\right)$ & $\operatorname{deg}\left(b_{3}\right)$ & $\operatorname{deg}\left(b_{4}\right)$ \\
\hline $\mathrm{sq}, L_{y}=4$ & 2 & 3 & 4 & 8 & 10 & 12 & - & 4 & 6 & 8 & - \\
\hline hc, $L_{y}=3$ & 2 & 3 & 6 & 10 & 12 & 10 & - & 6 & 8 & 6 & - \\
\hline$s q_{d}, d=4$ & 2 & 3 & 6 & 8 & 10 & 12 & - & 6 & 8 & 10 & - \\
\hline $\operatorname{tri}, L_{y}=4$ & 3 & 4 & 4 & 8 & 10 & 12 & 14 & 4 & 6 & 8 & 10 \\
\hline$\left(4 \cdot 8^{2}\right) L_{y}=3$ & 2 & 3 & 12 & 16 & 20 & 18 & - & 12 & 16 & 18 & - \\
\hline
\end{tabular}


We next list the polynomials $a_{s, j}$ and $b_{s, k}$ that we have calculated for several strip graphs $G_{s}$. Others are listed in Appendix 2.

\section{A. Strip of the Square Lattice}

For strips of the square lattice of width $L_{y}$, we take the repeating subgraph unit to be a vertical stack of $w=L_{y}-1$ squares, and the initial subgraph $I$ on the right to be identical to this repeating unit, $I=H$. In illustrating the proof of Theorem 1, we have already discussed in detail our calculation of the generating function for the square strip of width $L_{y}=3$ and have listed the resultant polynomials $a_{s q(3), 0}, a_{s q(3), 1}$, $b_{s q(3), 1}$, and $b_{s q(3), 2}$ as eqs. (2.40), (2.41), (2.35), and (2.36). For the square strip of width $L_{y}=4$, we find $j_{\max }=2, k_{\max }=3$, and, (with the notation $G_{s q\left(L_{y}=4\right)} \equiv G_{s q(4)}$ )

$$
\begin{gathered}
a_{s q(4), 0}=q(q-1) D_{4}(q)^{3} \\
a_{s q(4), 1}=-q(q-1)^{3}\left(q^{2}-4 q+5\right)\left(2 q^{4}-15 q^{3}+45 q^{2}-65 q+40\right) \\
a_{s q(4), 2}=q(q-1)^{3}\left(q^{8}-16 q^{7}+112 q^{6}-449 q^{5}+\right. \\
\left.1130 q^{4}-1829 q^{3}+1858 q^{2}-1084 q+279\right) \\
b_{s q(4), 1}=-q^{4}+7 q^{3}-23 q^{2}+41 q-33 \\
b_{s q(4), 2}=2 q^{6}-23 q^{5}+116 q^{4}-329 q^{3}+553 q^{2}-517 q+207 \\
b_{s q(4), 3}=-q^{8}+16 q^{7}-112 q^{6}+449 q^{5}-1130 q^{4}+1829 q^{3}-1858 q^{2}+1084 q-279
\end{gathered}
$$

\section{B. Strip of the Triangular Lattice}

We consider a strip of the triangular lattice of width $L_{y}$. The $H$ and $I$ subgraphs were defined above in section II. The strip graph of this type with $m$ repeated $H$ subgraph units is denoted $\left(G_{t\left(L_{y}\right)}\right)_{m}$. The case $L_{y}=2$ yields a chromatic polynomial that factorizes, as in eq. (2.15). For $L_{y}=3$ we find $j_{\max }=1$ and $k_{\max }=2$ with

$$
\begin{gathered}
a_{t(3), 0}=q(q-1)(q-2)^{4} \\
a_{t(3), 1}=-q(q-1)^{2}(q-2)^{3}(q-3) \\
b_{t(3), 1}=-q^{3}+7 q^{2}-18 q+17 \\
b_{t(3), 2}=(q-2)^{3}(q-3)
\end{gathered}
$$

The results for the next wider strip, with $L_{y}=4$, are somewhat lengthy and are given in Appendix 2 . 


\section{Strip of the Honeycomb Lattice}

The minimal-width strip for the honeycomb (also called hexagonal) lattice with a nonfactorizing chromatic polynomial is shown in Fig. 1(e) and has a width $L_{y}=3$ (represented as a brick lattice). In contrast to the strips of the square and triangular lattice with the same $L_{y}=3$ width, the honeycomb strip yields a generating function with numerator and denominator functions that are of higher degrees in $x, v i z ., j_{\max }=2$ and $k_{\max }=3$. We find

$$
\begin{gathered}
a_{h c(3), 0}=q(q-1) D_{6}(q)^{2} \\
a_{h c(3), 1}=-q(q-1)^{2}\left(q^{9}-13 q^{8}+76 q^{7}-262 q^{6}+589 q^{5}\right. \\
\left.-901 q^{4}+943 q^{3}-651 q^{2}+266 q-47\right) \\
a_{h c(3), 2}=q(q-1)^{7}(q-2)^{2} \\
b_{h c(3), 1}=-q^{6}+8 q^{5}-28 q^{4}+56 q^{3}-71 q^{2}+58 q-26 \\
b_{h c(3), 2}=(q-1)^{2}\left(q^{6}-10 q^{5}+43 q^{4}-102 q^{3}+144 q^{2}-120 q+49\right) \\
b_{h c(3), 3}=-(q-1)^{4}(q-2)^{2}
\end{gathered}
$$

\section{Strip of the Kagomé Lattice}

It is also of interest to calculate generating functions for the chromatic polynomials of strips of lattices which involve more than just one regular polygons. The uniform lattices that involve tilings of the plane by one or more regular polygons are called Archimedean (for a review, see Ref. [31]); for some related studies, see Refs. 22, 24, 32 ) and, since each vertex is equivalent to every other, these lattices may be defined by the sequence of polygons that one traverses when making a circuit (say, in a counterclockwise direction) around any vertex,

$$
\Lambda=\prod_{i} p_{i}^{a_{i}}
$$

where $p_{i}$ label the polygon and $a_{i} \geq 1$ is the number of times that a given polygon occurs contiguously in the product. The total number of times that a polygon of type $p_{i}$ occurs is the sum of its contiguous and noncontiguous appearances. The Archimedean lattices include three homopolygonal (also called monohedral) cases: square, triangular, and honeycomb. The remaining Archimedean lattices are heteropolygonal, i.e., 
are composed of more than one type of regular polygon. For the strips of heteropolygonal lattices, it is necessary to fix a convention to describe the width. We shall choose the convention of taking the width to be the number of layers of the polygon with the greatest number of sides, and, to retain a correspondence with the homopolygonal lattices, we shall label the polynomials in $\Gamma\left(G_{s}, q, x\right)$ by $w+1$ (which is $L_{y}$ for the homopolygonal strips). For our present study we consider first a strip of the kagomé lattice, denoted $(3 \cdot 6 \cdot 3 \cdot 6)$ in the standard mathematical notation (3.17). We take the strip to be of width $w=1$ and oriented as shown in Fig. 1 1 (f). The initial subgraph $I$ on the right is a hexagon, and the repeating subgraph unit $H$ is the graph comprised of a hexagon and the two triangles adjacent to it. We find $j_{\max }=1, k_{\max }=2$ and

$$
\begin{gathered}
a_{\text {kag }(2), 0}=q(q-1) D_{6}(q) \\
a_{k a g(2), 1}=-q(q-1)^{5}(q-2) \\
b_{k a g(2), 1}=-(q-2)\left(q^{4}-6 q^{3}+14 q^{2}-16 q+10\right) \\
b_{\text {kag }(2), 2}=(q-1)^{3}(q-2)^{3}
\end{gathered}
$$

We have also calculated generating functions for two other heteropolygonal Archimedean lattices, viz., the $\left(3 \cdot 12^{2}\right)$ and $\left(4 \cdot 8^{2}\right)$ lattices. The results for these are given in Appendix 2. We next proceed to study the limit in which the length of a strip goes to infinity.

\section{ANALYTIC STRUCTURE OF $W\left(\left\{G_{s}\right\}, q\right)$}

To calculate $W\left(\left\{G_{s}\right\}, q\right)$ from the generating function $\Gamma\left(G_{s}, q, x\right)$, we prove a basic theorem. Let us write the denominator function $\mathcal{D}$ of the generating function $\Gamma\left(G_{s}, q, x\right)$ in factorized form, in terms of its roots $r_{s, k}, k=1, \ldots, k_{\max }$, as

$$
\mathcal{D}\left(G_{s}, q, x\right)=1+\sum_{k=1}^{k_{\max }} b_{s, k}(q) x^{k}=b_{s, k_{\max }} \prod_{k=1}^{k_{\max }}\left(x-r_{s, k}(q)\right)
$$

or equivalently

$$
\mathcal{D}\left(G_{s}, q, x\right)=\prod_{k=1}^{k_{\max }}\left(1-\lambda_{s, k}(q) x\right)
$$

where

$$
\lambda_{s, k}(q)=\frac{1}{r_{s, k}(q)}
$$

(and the roots satisfy the relation $b_{s, k_{\max }}(-1)^{k_{\max }} \prod_{k=1}^{k_{\max }} r_{s, k}=1$.) 


\section{Theorem 3}

Consider a strip graph $G_{s}$ as defined in eq. (1.2), constructed by $m$ successive additions of a repeating graphical subunit $H$ to an initial subgraph $I$. For a given value of $q$, denote the function $\lambda_{s, k}(q)$ of maximal magnitude in eq. (4.2) as $\lambda_{s, \max }(q)$. Then the $n \rightarrow \infty$ asymptotic limiting function $W\left(\left\{G_{s}\right\}, q\right)$ as defined in eq. (1.1) with eq. (1.3) is given by

$$
W\left(\left\{G_{s}\right\}, q\right)=\left(\lambda_{s, \max }(q)\right)^{1 / c_{s, 1}}
$$

where the definition of $c_{s, 1}$ was given in eq. (2.2) and a general formula for it was presented in eq. (2.4). The phase to pick in evaluating eq. (1.5) and eq. (4.4) is chosen so that for sufficiently large real $q, W\left(\left\{G_{s}\right\}, q\right)$ is real.

Proof

First, for the case $k_{\max }=1$, where the chromatic polynomial for the strip factorizes according to eq. (2.15), one recognizes

$$
\lambda_{s}(q)=-b_{s, 1}(q)=P_{r}(H, q)
$$

where $P_{r}(H, q)$ was given in eq. (2.13), and the result (4.4) then follows immediately from the elementary expansion

$$
\Gamma\left(G_{s}, q, x\right)=\frac{\mathcal{N}\left(G_{s}, q, x\right)}{1-\lambda_{s}(q) x}=\mathcal{N}\left(G_{s}, q, x\right) \sum_{m=0}^{\infty} \lambda_{s}(q)^{m} x^{m}
$$

together with eqs. (2.9) and (1.1), whence

$$
W\left(\left\{G_{s}\right\}, q\right)=\lim _{n \rightarrow \infty}\left(\lambda_{s}(q)\right)^{m / n}=\left(\lambda_{s}(q)\right)^{1 / c_{s, 1}}
$$

where we have used eq. (2.2). Note that, for the generic case where $c_{s, 1} \geq 2, W\left(\left\{G_{s}\right\}, q\right)$ has branch point singularities at the zeros (if any) of $\lambda_{s}(q)$. We shall next discuss the case $k_{\max }=2$ and comment on the straightforward generalization to $k_{\max } \geq 3$. For brevity of notation, we shall suppress the $q$-dependence of the $\lambda_{s, k}(q)$. For $k_{\max }=2$, eq. (2.10) becomes

$$
\begin{aligned}
\Gamma\left(G_{s}, q, x\right) & =\frac{\mathcal{N}\left(G_{s}, q, x\right)}{\left(1-\lambda_{s, 1} x\right)\left(1-\lambda_{s, 2} x\right)}=\frac{\mathcal{N}\left(G_{s}, q, x\right)}{\left(\lambda_{s, 1}-\lambda_{s, 2}\right)}\left[\frac{\lambda_{s, 1}}{1-\lambda_{s, 1} x}-\frac{\lambda_{s, 2}}{1-\lambda_{s, 2} x}\right] \\
& =\frac{\mathcal{N}\left(G_{s}, q, x\right)}{\left(\lambda_{s, 1}-\lambda_{s, 2}\right)} \sum_{m=0}^{\infty}\left(\lambda_{s, 1}^{m+1}-\lambda_{s, 2}^{m+1}\right) x^{m}
\end{aligned}
$$

(which is, of course, symmetric under $\lambda_{s, 1} \leftrightarrow \lambda_{s, 2} \cdot$ ) Then, from the definition (1.1) with (1.3), taking the $1 / n$ 'th power as $n \rightarrow \infty$, and again using eq. (2.2), we obtain the result (4.4). It is straightforward to 
generalize this to the case $k_{\max } \geq 3$. In passing, we note that if some fixed subset $N_{e q} \geq 2$ of the $\lambda_{s, k}$ were identically equal, this would not affect the result 4.4). (This is similar to the situation where some number of the eigenvalues of a transfer matrix for a spin model are identically equal [33.) This is trivial if the identical $\lambda_{s, k}$ 's are not maximal; if they are, it is also clear from the fact that

$$
\lim _{n \rightarrow \infty}\left(N_{e q}\left(\lambda_{s, \max }(q)\right)^{m+1}\right)^{1 / n}=\left(\lambda_{s, \max }(q)\right)^{1 / c_{s, 1}}
$$

independent of $N_{e q}$. (This possibility is mentioned only for completeness; we have not encountered any strip graph $G_{s}$ where any $\lambda_{s, k}(q)$ are identically equal.) The choice of phase, $r=0$ in eq. (1.5) is determined by the fact that for sufficiently large real $q, \lambda_{s, \max }(q)>0$.

This completes the proof.

We next state some corollaries to this theorem.

\section{Corollary 3.1}

Given the strip graph $G_{s}$ as defined in eq. (1.2), the asymptotic function $W\left(\left\{G_{s}\right\}, q\right)$ as defined in (1.1) with $(1.3)$ is independent of the numerator function $\mathcal{N}\left(G_{s}, q, x\right)$. It follows also, a fortiori, that the continuous locus of points $\mathcal{B}$ where $W\left(\left\{G_{s}\right\}, q\right)$ is nonanalytic, is independent of $\mathcal{N}\left(G_{s}, q, x\right)$.

\section{Corollary 3.2}

Given the strip graph $G_{s}$ as defined in eq. (1.2), the asymptotic function $W\left(\left\{G_{s}\right\}, q\right)$ as defined in (1.1) with (1.3) is independent of the initial subgraph $I$ and depends only on the infinitely repeated subgraph unit $H$. Furthermore, a fortiori, the continuous locus of points $\mathcal{B}$ where $W\left(\left\{G_{s}\right\}, q\right)$ is nonanalytic is independent of $I$ and depends only on $H$.

In particular, for this second corollary, recall that our proof of Theorem 1 showed that $\mathcal{D}$ was independent of the initial subgraphs of the strip graphs generated by the operation of the contraction-deletion theorem, as was noted before eq. (2.33). It is interesting to see Corollaries 3.1 and 3.2 in operation by studying the chromatic zeros of strip graphs of a given type $G_{s}$, varying the type of initial subgraph $I$ and the number of repeated subgraph units $m$. For small $m$, we find, as expected, that the chromatic zeros clearly depend on what $I$ one uses, but as $m$ gets larger and larger, this dependence is progressively reduced, until it disappears altogether as $m \rightarrow \infty$. Moreover, as we shall discuss in later work [29], for strip graphs of the form $J\left(\prod_{\ell=1}^{m} H\right) I$, the asymptotic $m \rightarrow \infty$ limiting functions $W\left(\left\{G_{s}\right\}, q\right)$ do, in general, depend on both of the end graphs $I$ and $J$.

For a strip graph $\left(G_{s}\right)_{m}$ composed of $m$ repeated subgraph units $H$ adjoined to an initial subgraph $I=H$ and having the property that the chromatic polynomial factorizes as in (2.15), the asymptotic limiting function is given by 


$$
W\left(\left\{G_{s ; K_{\ell}}\right\}, q\right)=\left(P_{r}(H, q)\right)^{1 /(n(H)-\ell)}
$$

where we have used eq. (2.4) for $c_{s, 1}$. For the proof of this result, we observe that from the formula (2.16), one can immediately identify $\lambda_{s}(q)=P_{r}(H, q)$, and eq. (4.10) then follows from Theorem 3 and its special case eq. (4.7) for $k_{\max }=1$. For example, for strips of the square and triangular lattices of width $L_{y}=2$, using the $\ell=2$ special case of eq. (4.10), we obtain

$$
W\left(\left\{G_{s q(2)}\right\}, q\right)=\left(q^{2}-3 q+3\right)^{1 / 2}
$$

and

$$
W\left(\left\{G_{t(2)}\right\}, q\right)=q-2
$$

For the case $k_{\max }=2$, the roots of $\mathcal{D}\left(G_{s}, q, x\right)=0$ as an equation in $x$ are $r_{s, k}, k=1,2$, given by $r_{s, \pm}=\left(-b_{s, 1} \pm \sqrt{R_{s}}\right) /\left(2 b_{s, 2}\right)$, so that the $\lambda_{s, k}, k=1,2$, are

$$
\lambda_{s, \pm}=r_{s, \pm}^{-1}=-\frac{1}{2}\left[b_{s, 1} \pm \sqrt{R_{s}}\right]
$$

where the discriminant $R_{s}$ is

$$
R_{s}=b_{s, 1}^{2}-4 b_{s, 2}
$$

Corollary 3.3

Given the strip graph $G_{s}$ as defined in eq. (1.2), the maximal $\lambda_{s, \max }(q)$ in eqs. (4.2) and (4.4) has the behavior, for large real $q$,

$$
\lambda_{s, \max }(q) \sim q^{c_{s, 1}} \quad \text { as } \quad q \rightarrow \infty
$$

Proof

A chromatic polynomial for an arbitrary $n$-vertex graph $G$ is of degree $n$ as a polynomial in $q$ (with highest-degree term is $\left.q^{n}\right)$. Hence, from the definition (1.1), it follows that $W(\{G\}, q) \sim q$ as $q \rightarrow \infty$ for real $q$ (more generally, $|W(\{G\}, q)| \sim|q|$ for complex $q$ ). Combining this with Theorem 3, eq. (4.4) yields the result (4.15).

We next prove a theorem which explicitly determines the continuous locus of points $\mathcal{B}$ where $W\left(\left\{G_{s}\right\}, q\right)$ is nonanalytic:

Theorem 4 
Let a strip graph $G_{s}$ be defined as in eq. (1.2). Then the continuous locus of points $\mathcal{B}$ where the asymptotic function $W\left(\left\{G_{s}\right\}, q\right)$ is nonanalytic is comprised of the points where two (nonzero) $\lambda_{s, k}$ terms in eq. (4.2) both become maximal and degenerate in magnitude, i.e., the solutions to the equation

$$
\left|\lambda_{s, \max }(q)\right|=\left|\lambda_{s, \max ^{\prime}}(q)\right|
$$

Proof

This follows from the general analysis of $W(\{G\}, q)$, specifically part (d) of Theorem 1, given in Ref. [21]. Note that, from eq. (4.4), if $c_{s, 1} \geq 2$, then $W\left(\left\{G_{s}\right\}, q\right)$ has isolated branch point singularities where $\lambda_{s, \max }(q)=0$, but, in general, these do not lie on the continuous locus $\mathcal{B} . \quad \square$.

A simple corollary to this theorem is that if the strip graph $G_{s}$ defined by eq. (1.2) has the property that its chromatic polynomial factorizes in the form of eq. (2.15), then $W\left(\left\{G_{s}\right\}, q\right)$, which we have calculated in eq. (4.10), is analytic in the complex $q$ plane, except for isolated branch points, so that $\mathcal{B}$ is the null set, $\emptyset$. This follows because in this case there is only one $\lambda_{s}$, (recall eq. (4.6)) and hence no possibility for a degeneracy of maximal $\lambda_{s, k}$ 's or resultant locus $\mathcal{B}$. For example, in the case where $H$ is a $p$-sided polygon and each successive $p$-gon intersects the next on a mutual edge so that $L_{H}=K_{2}$, we have

$$
W\left(\left\{G_{p-g o n s ; K_{2}}\right\}, q\right)=\left(D_{p}(q)\right)^{1 /(p-2)}
$$

where $D_{p}(q)$ was defined in eq. (2.18). The continuous locus $\mathcal{B}=\emptyset$ for these graphs. For $p \geq 4$, the function $W\left(\left\{G_{p-\text { gons } ; K_{2}}\right\}, q\right)$ has isolated branch point singularities at the $p-2$ zeros of the polynomial $D_{p}(q)$, but these are not connected with any continuous locus of singularities $\mathcal{B}$. Note that $D_{p}(q)$ is a polynomial of degree $p-2$; for odd $p \geq 3, D_{p}(q)$ has a single real zero at $q=2$ and $(p-1) / 2$ conjugate pairs of complex zeros, while for even $q, D_{p}(q)$ has only complex zeros.

Before proceeding to discuss $W\left(\left\{G_{s}\right\}, q\right)$ for specific strip graphs $G_{s}$, we recall that the elementary property that for any family of graphs $\{G\}$, the associated locus of points $\mathcal{B}$ is invariant under complex conjugation:

$$
\mathcal{B}(q)=\mathcal{B}\left(q^{*}\right)
$$

This follows from the fact that the coefficients of each power of $q$ in a chromatic polynomial $P(G, q)$ are real (indeed, integers) and the fact that $\mathcal{B}$ originates as a merging of zeros of $P(G, q)$ as the number of vertices $n \rightarrow \infty$ [21.

\section{V. $W\left(\left\{G_{s}\right\}, q\right)$ FOR SPECIFIC STRIP GRAPHS}

We next apply our general theorems to calculate the exact $W\left(\left\{G_{s}\right\}, q\right)$ functions for specific infinitely long strip graphs. The continuous locus of points $\mathcal{B}$ where $W\left(\left\{G_{s}\right\}, q\right)$ is nonanalytic is shown in each of the accompanying figures. As was discussed above, as the length of the strip graph goes to infinity, the 
locus $\mathcal{B}$ forms by the merging together of the zeros of the chromatic polynomial (i.e., the chromatic zeros) of this strip graph. One gains insight into how this limit is approached by calculating chromatic zeros for reasonably long finite strips of each type. Accordingly, we show these in the figures. There are also isolated chromatic zeros that occur at $q=q_{s}=0,1$, and, for graphs that contain one or more triangles, at $q_{s}=2$. As discussed in Ref. [21] and the Introduction, in cases (i) where these occur via factors that scale like the lattice size, i.e., $\left(q-q_{s}\right)^{t n}, t \geq 1$, they remain as zeros of the limiting function $W(\{G\}, q)$; (ii) if, on the other hand, they occur as factors like $\left(q-q_{s}\right)^{u}$ where $u$ does not scale like $n$, then they do not appear in $W(\{G\}, q)$. (If one were to use the alternate definition based on the opposite order of limits in (1.4), then these zeros at $q=q_{s}$ would appear as discontinuities in $W\left(\left\{G_{s}\right\}, q\right)$.) In case (ii) the different orders of limits in eq. (1.4) do not commute, whereas in case (i) they do. Depending on the type of strip graph, isolated real chromatic zeros may occur at certain other points also. For example, in Fig. B(b) there is a chromatic zero near to, but not precisely at, $q=2$ and another at $q \simeq 2.239$, while in Fig. E(b) there is a chromatic zero at $q=(3+\sqrt{5}) / 2=2.6180 \ldots$, etc. The simplest types of strip graphs to consider are those with chromatic polynomials that factorize in the manner of eq. (2.15), corresponding to $k_{\max }=1$; we have already discussed these above. 

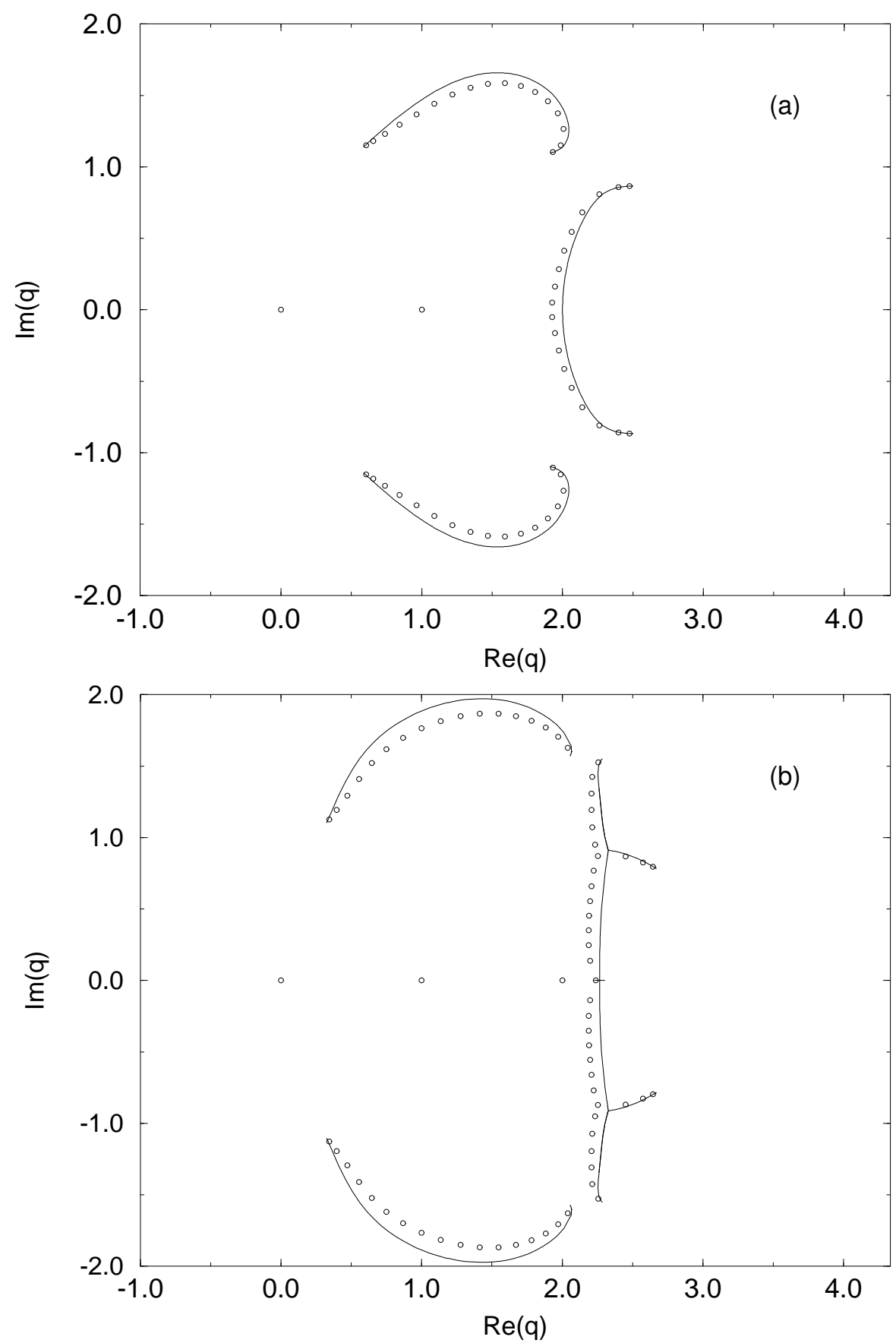
FIG. 3. Analytic structure of the function $W\left(\left\{G_{s q\left(L_{y}\right)}\right\}, q\right)$ for $L_{y}=$ (a) 3, (b) 4 , where $\left\{G_{s q\left(L_{y}\right)}\right\}$ denotes the $\left(L_{x}=\infty\right) \times L_{y}$ strip of the square lattice. The arcs are the continuous locus of points $\mathcal{B}$ where this function is nonanalytic. For comparison, the zeros of the chromatic polynomial $P\left(\left(G_{s q\left(L_{y}\right)}\right)_{m}, q\right)$ for (a) $L_{y}=3, m=16$ (hence $n=54$ vertices) and (b) $L_{y}=4, m=16$ (hence $n=72$ ) are shown. In this and subsequent figures, the initial subgraph $I$ is specified in the text.

We proceed to consider strips graphs with denominator functions with $k_{\max }=2$, i.e., $\mathcal{D}(x)$ that are quadratic in $x$ (listed in Table 1). For these, we have the explicit results (4.13), (4.14). We first consider the strip graph of the square lattice of size $L_{x} \times L_{y}=\infty \times 3$. (As was noted at the beginning, we always use free boundary conditions in this paper.) From eqs. (2.35) and (2.36), we calculate the $W$ function by starting on the real axis with $q>2$ and analytically continuing from there. In this range, $b_{s q(3), 1}<0$ and so $\lambda_{s q(3), \max }=\lambda_{s q(3),-}$, where $\lambda_{s, \pm}$ was given in eq. (4.13). Hence, in this range,

$$
\begin{aligned}
W\left(\left\{G_{s q(3)}\right\}, q\right)= & 2^{-1 / 3}\left[(q-2)\left(q^{2}-3 q+5\right)+\right. \\
& {\left.\left[\left(q^{2}-5 q+7\right)\left(q^{4}-5 q^{3}+11 q^{2}-12 q+8\right)\right]^{1 / 2}\right]^{1 / 3} }
\end{aligned}
$$

In Fig. 3(a) we show our calculation of the exact continuous locus of points $\mathcal{B}$ where $W\left(\left\{G_{s q(3)}\right\}, q\right)$ is nonanalytic. These consist of two complex-conjugate (c.c.) arcs together with a self-conjugate arc that crosses the real axis at $q=2$, where $b_{s q(3), 1}$ vanishes. Since $\mathcal{B}$ does not enclose any regions, we can analytically continue the expression (5.1) throughout the $q$ plane, exclusive of the points on the locus $\mathcal{B}$ itself. The two complex zeros of $b_{s q(3), 1}$ at $\left.q=3 / 2 \pm(i / 2) \sqrt{11}\right)=1.5 \pm 1.66 i$ lie on the upper and lower arcs. The discriminant of the square root in eq. (5.1) has zeros at $q=5 / 2 \pm(i / 2) \sqrt{3}$ (for the quadratic factor) and $q=0.587 \pm 1.14 i$ and $1.91 \pm 1.10 i$; (for the quartic factor). These zeros are all simple and hence are branch points of the square root. As is evident in Fig. 3(a), the first pair form the endpoints of the self-conjugate arc crossing the real axis, while the second two pairs form the endpoints of the two complex-conjugate arcs. In Fig. 3(a) we also show our calculations of the chromatic zeros for a long finite strip of the square lattice As expected, aside from the generally occurring isolated zeros at $q=0$ and 1 , these zeros lie close to the arcs that comprise $\mathcal{B}$ in the infinite-length limit.

Next, in Fig. 3(b) we present our exact calculation for $\mathcal{B}$ for a square strip of the next greater width, i.e., size $L_{x} \times L_{y}=\infty \times 4$, together with chromatic zeros computed for a long finite strip. Our explicit analytic expression for $W\left(\left\{G_{s q(4)}\right\}, q\right)$, which involves the root of a cubic equation, is rather complicated and hence is not given here; it can be obtained from eqs. (3.4)-(3.6). The comparison of the results presented in Fig. 3(b) with those for the $L_{y}=3$ strip in Fig. $3($ a) shows how this locus $\mathcal{B}$ changes as the width of a strip of a given

\footnotetext{
${ }^{5}$ We recall the well-known fact that calculations of the zeros of chromatic polynomials are difficult because for a graph $G$ with $n$ vertices, the coefficients $p_{\ell}$ in the chromatic polynomial $P(G, q)=\sum_{\ell=1}^{n} p_{n} q^{n}$ range from $p_{n}=1$ to much larger values for coefficients of terms $q^{\ell}$ with $\ell \simeq n / 2$, as is clear from the theorem $[16]$ that $\left|p_{r}\right| \geq\left(\begin{array}{l}n-1 \\ r-1\end{array}\right)$. For this reason, it is necessary to use a large number of digits of accuracy in routines to solve for the roots.
} 
type increases. We see that all three of the arcs elongate, and the single self-conjugate arc of the $L_{y}=3$ strip develops a more complicated structure for the $L_{y}=4$ strip. In particular, multiple points appear on this arc for $L_{y}=4$. Here, we use the term "multiple point" in the technical sense of algebraic geometry, meaning a point on an algebraic curve where two or more branches of the curve meet 34.32]. The index of the multiple point is the number of branches that meet; in Fig. 3(b) there is (i) a multiple point at $q \simeq 2.265$ where a vertical component of $\mathcal{B}$ intersects a small line segment in $\mathcal{B}$ on the real axis, and (ii) two multiple points forming a complex-conjugate pair, at which three components of $\mathcal{B}$ meet. It is also interesting to compare the exact arcs that we have calculated for infinite strips, and the associated chromatic zeros for finite strips, with the chromatic zeros calculated in Ref. [20] for an $L_{x} \times L_{y}=8 \times 8$ square lattice with the same (free) boundary conditions. Already for a width $L_{y}=4$, the chromatic zeros for the strip resemble those of the larger patch of the $2 \mathrm{D}$ square lattice. There are branchings in the chromatic zeros for the $8 \times 8$ lattice at complex-conjugate points close to the locations where we find multiple points on $\mathcal{B}$ for the $\infty \times 4$ strip. Our previous results on $W(\{G\}, q)$ in Ref. [21] have shown that boundary conditions can strongly affect $\mathcal{B}$ and the associated chromatic zeros that merge to form this locus in the $n \rightarrow \infty$ limit of an $n$-vertex graph. For example, $\mathcal{B}=\emptyset$ for the infinite limit of a linear graph with free boundary conditions, whereas for the same graph with periodic boundary conditions, i.e., the infinite- $n$ limit of the circuit graph, we found that $\mathcal{B}$ is the circle $|q-1|=1$, which separates the complex $q$ plane into two distinct regions ${ }^{6}$. Nevertheless, it is intriguing to compare our results with the chromatic zeros computed for an $8 \times 8$ lattice with cylindrical boundary conditions, i.e., periodic in one direction and free in the other [20]. We observe that there are cusp-like structures in the chromatic zeros on the $8 \times 8$ lattice in the complex-conjugate regions where there are gaps between the arcs on the $\infty \times 4$ strip.

Using our results for $W\left(\left\{G_{s q\left(L_{y}\right)}\right\}, q\right)$, we can calculate the large- $q$ series for the reduced functions $W_{r}\left(\left\{G_{s q\left(L_{y}\right)}\right\}, q\right)$ defined for a general graph $G$ in eq. (2.51). In order to compare with the large- $q$ series expansion for the infinite square lattice, we actually consider the function $\bar{W}\left(\left\{G_{s q\left(L_{y}\right)}\right\}, q\right)$ defined in eq. (2.52). (For this purpose, we formally substitute $\zeta=4$, although not all of the vertices of the strip graphs have the same degree.) With $y=1 /(q-1)$ as in eq. (2.53), we find $\bar{W}\left(\left\{G_{s q(1)}\right\}, q\right)=1+y$, $\bar{W}\left(\left\{G_{s q(2)}\right\}, q\right)=(1+y)\left(1-y+y^{2}\right)^{1 / 2}$, whence

$$
\begin{aligned}
\bar{W}\left(\left\{G_{s q(2)}\right\}, q\right) & =1+\frac{1}{2} y-\frac{1}{2^{3}} y^{2}+\frac{9}{2^{4}} y^{3}+\frac{27}{2^{7}} y^{4}-\frac{9}{2^{8}} y^{5}-\frac{117}{2^{10}} y^{6}-\frac{135}{2^{11}} y^{7}+O\left(y^{8}\right) \\
& \simeq 1+0.5 y-0.125 y^{2}+0.5625 y^{3}+0.2109 y^{4}
\end{aligned}
$$

\footnotetext{
${ }^{6}$ In contrast, in statistical mechanics, the existence of the thermodynamic limit entails independence of boundary conditions, except in the well-understood sense that they can determine which of degenerate long range orderings the system takes in a low-temperature, broken-symmetry phase. This is one of several differences between the behavior of $W(\Lambda, q)$ and the somewhat analogous function for a spin model in statistical mechanics, $\exp (f(\Lambda, K))$, where $f$ is the per site free energy on a lattice $\Lambda$, and $K=\beta J$ as in the text. Another is the noncommutativity of eq. (1.4), which has no analogue in statistical mechanics. See Ref. 21] for further discussion.
} 


$$
-0.0352 y^{5}-0.1143 y^{6}-0.06592 y^{7}+O\left(y^{8}\right)
$$

and

$$
\begin{aligned}
\bar{W}\left(\left\{G_{s q(3)}\right\}, q\right)= & 1+\frac{1}{3} y-\frac{1}{3^{2}} y^{2}+\frac{59}{3^{4}} y^{3}+\frac{44}{3^{5}} y^{4}-\frac{32}{3^{6}} y^{5}-\frac{613}{3^{8}} y^{6}+\frac{5666}{3^{9}} y^{7}+O\left(y^{8}\right) \\
\simeq & 1+0.333 y-0.111 y^{2}+0.728 y^{3}+0.181 y^{4} \\
& -0.044 y^{5}-0.093 y^{6}+0.288 y^{7}+O\left(y^{8}\right)
\end{aligned}
$$

These may be compared with the first few terms of the series for the infinite lattice [30]

$$
\bar{W}(s q, q)=1+y^{3}+y^{7}+O\left(y^{8}\right)
$$

We see that as the width of the strip increases, the coefficients in the small-y series expansion of the corresponding $\bar{W}$ function approach those of the infinite lattice, up to terms of order $O\left(y^{p}\right)$, where $p \sim L_{y}$. In particular, the coefficients of the terms $y^{k}, k=1,2,4,5,6$ are smaller than those of the $y^{3}$ and $y^{7}$ terms. Evidently, as $L_{y} \rightarrow \infty$, these smaller coefficients eventually decrease to zero, while the coefficients of the $y^{3}$ and $y^{7}$ terms increase to their infinite-lattice values of unity. For progressively larger strips, similar behavior occurs for the coefficients of higher terms in the small-y series. In this way one can see from small- $y$ series expansions of $\bar{W}$ functions on infinitely long strips how the infinite-lattice series arise.

It is also of interest to see how the values of the $W\left(\left\{G_{s q\left(L_{y}\right)}\right\}, q\right)$ functions for the strips of various widths $L_{y}$ compare with those of the infinite square lattice, $W\left(\left\{G_{s q\left(L_{y}=\infty\right)}\right\}, q\right) \equiv W(s q, q)$ for various values of $q$. For example, for $q=3$, we have $W\left(\left\{G_{s q(1)}\right\}, 3\right)=2, W\left(\left\{G_{s q(2)}\right\}, 3\right)=\sqrt{3}=1.73205 \ldots, W\left(\left\{G_{s q(3)}\right\}, 3\right)=$ $[(1 / 2)(5+\sqrt{17})]^{1 / 3}=1.65846 \ldots$, and $W\left(\left\{G_{s q(4)}\right\}, 3\right)=1.624945 \ldots$ These decrease monotonically toward the infinite-lattice value [19] $W\left(\left\{G_{s q(\infty)}\right\}, 3\right) \equiv W(s q, 3)=(4 / 3)^{3 / 2}=1.53960 \ldots$. Defining the ratio

$$
R_{W}\left(\left\{G_{s q\left(L_{y}\right)}\right\}, q\right)=\frac{W\left(\left\{G_{s q\left(L_{y}\right)}\right\}, q\right)}{W\left(\left\{G_{s q(\infty)}\right\}, q\right)}
$$

we have $R_{W}\left(\left\{G_{s q(1)}\right\}, 3\right)=1.2990 \ldots, R_{W}\left(\left\{G_{s q(2)}\right\}, 3\right)=1.12500 \ldots, R_{W}\left(\left\{G_{s q(3)}\right\}, 3\right)=1.07720 \ldots$, and $R_{W}\left(\left\{G_{s q(4)}\right\}, 3\right)=1.05543 \ldots$. Thus the infinitely long strip graph of the square lattice with width $L_{y}=4$ already yields a $W$ function which is, for $q=3$, rather close to the value for the infinite square lattice. We have verified that this is also true for higher values of $q$, using our Monte Carlo measurements of $W(s q, q)$ in (Table 1 of) Ref. [21]. We have also verified that strips graphs of other lattices show similar behavior. We have found that, for a fixed $q \geq q_{c}(\Lambda), R_{W}\left(\left\{G_{s\left(L_{y}\right)}\right\}, q\right)$ decreases toward 1 as the strip width increases. Here, following our discussion in Refs. [21.22,24, $q_{c}(\Lambda)$ denotes the maximal (finite) real value of $q$ where $W(\Lambda, q)$ is nonanalytic; in the above works, we obtained the values $q_{c}(s q)=3, q_{c}(t)=4$, and $q_{c}(h c)=(3+\sqrt{5}) / 2=2.618$. for the square, triangular, and honeycomb lattices, respectively (see further discussion below on boundary conditions). 

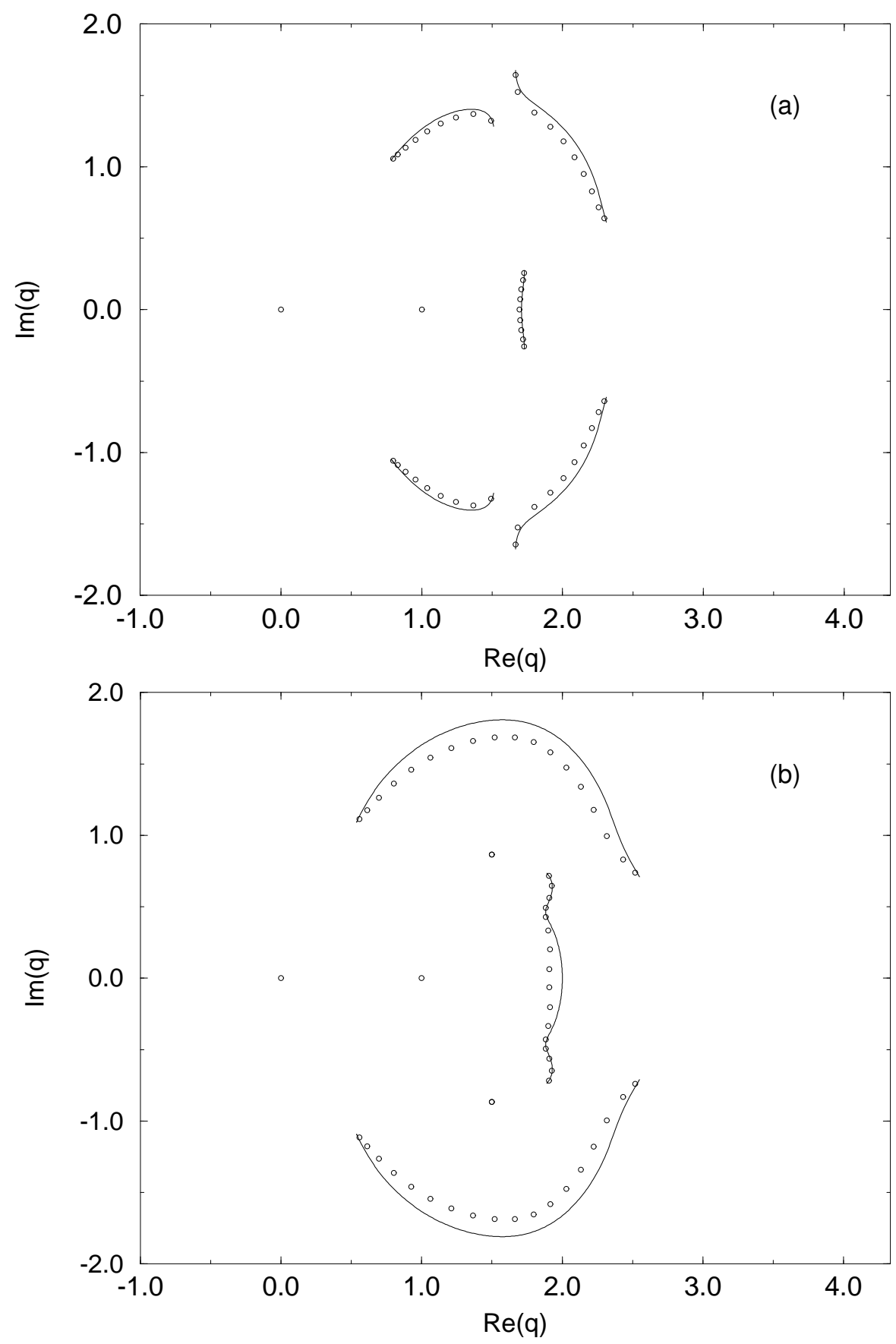
FIG. 4. Analytic structure of the function $W\left(\left\{G_{s q_{d}\left(L_{d}\right)}\right\}, q\right)$ for $L_{d}=(\mathrm{a}) 3$, (b) 4 , where $\left\{G_{s q_{d}\left(L_{d}\right)}\right\}$ denotes an infinitely long strip along a diagonal direction of the square lattice, for the case where the repeating subgraph $H$ has diagonal width $d=L_{d}$. Zeros of the chromatic polynomial $P\left(\left(G_{s q_{d}\left(L_{d}\right)}\right)_{m}, q\right)$ for (a) $L_{d}=3, m=9$ (so $n=49$ ) and (b) $L_{d}=4, m=8$ (so $n=56)$ are shown.

In Figs. 目(a) and $4(\mathrm{~b})$ we show our exact calculations of $\mathcal{B}$ for infinitely long strips of the square lattice along the diagonal direction. Finite strips of these two respective types were shown in Fig. 1 1 (c,d). Comparing the two different-width strips, one sees that the two pairs of complex-conjugate arcs in the narrower strip merge together to form a single such pair on the wider strip, while the self-conjugate arc elongates. Furthermore, from a comparison of these two figures with the previous two, one sees several similarities but also observes that even for a given lattice, the details of $\mathcal{B}$ depend, in general, on the orientation of the strip with respect to the principal lattice directions.

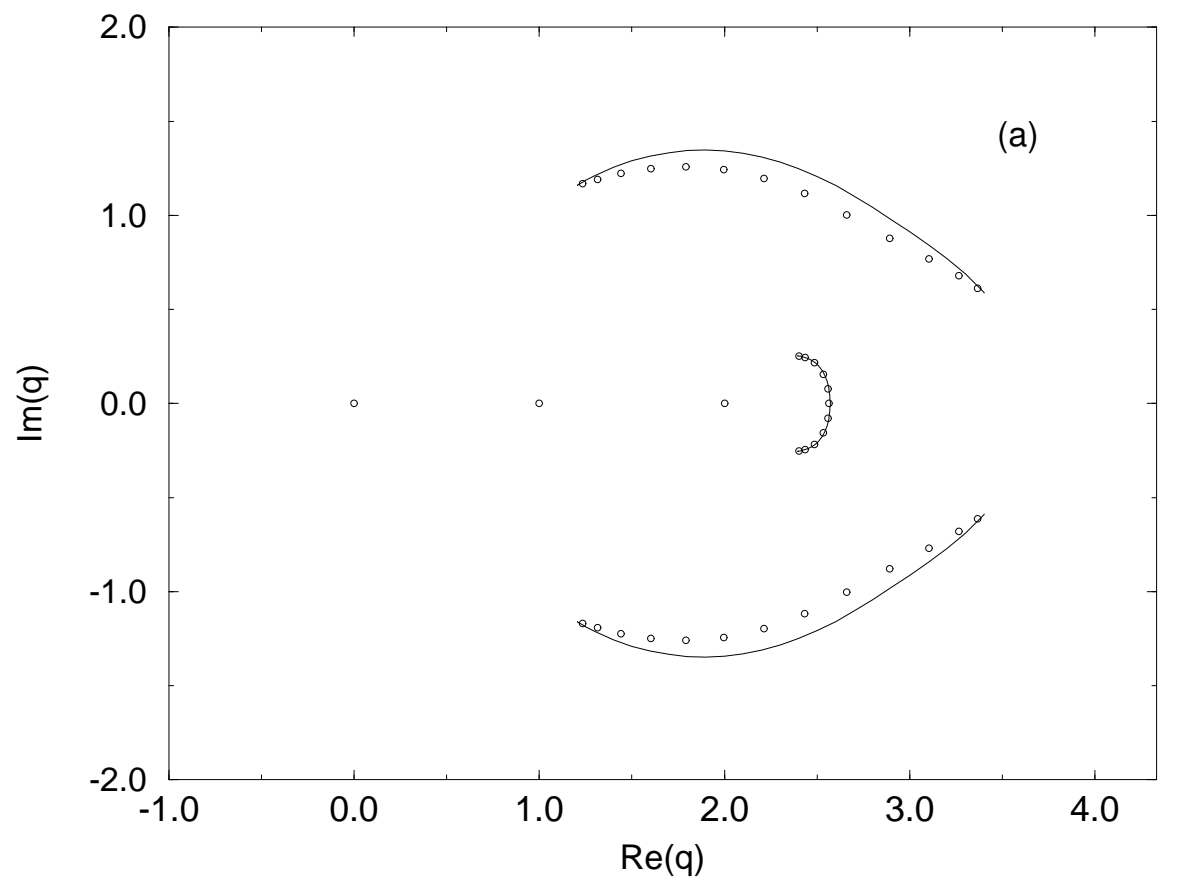




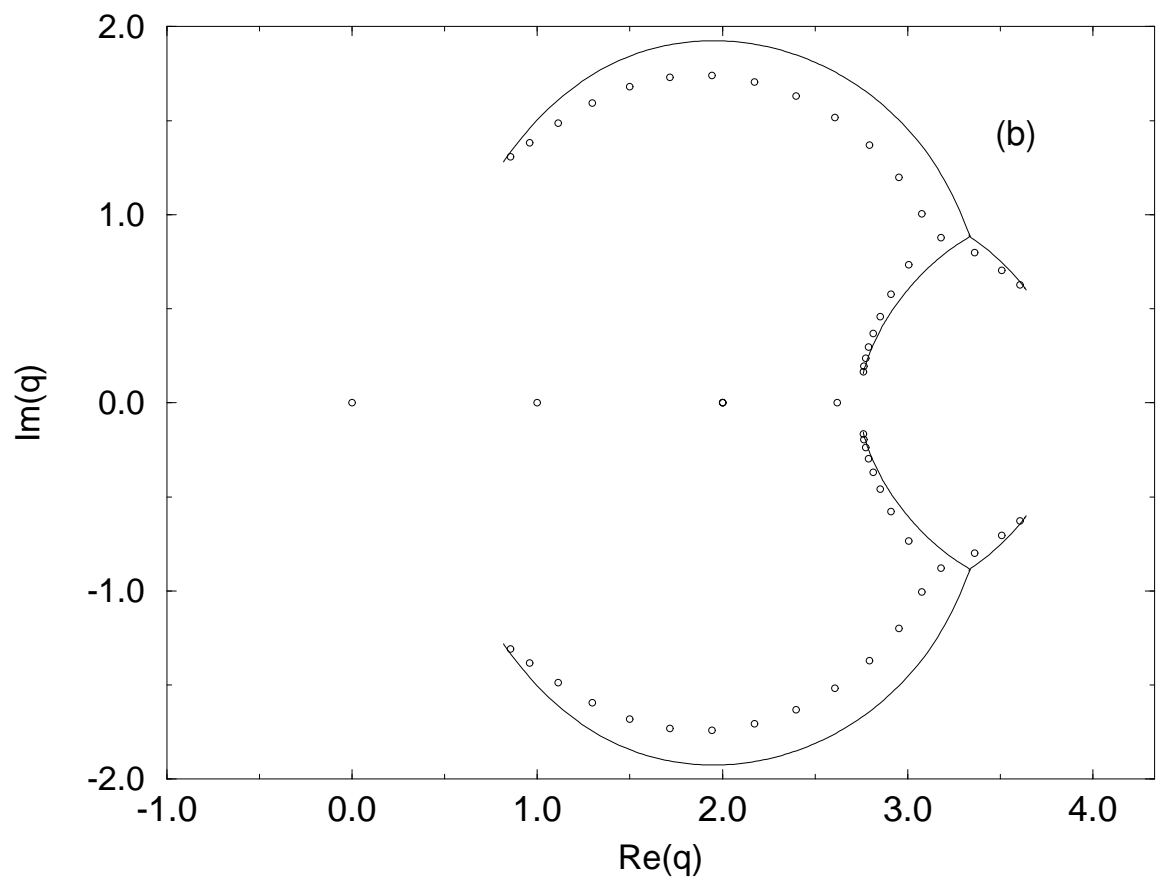

FIG. 5. Analytic structure of the function $W\left(\left\{G_{t\left(L_{y}\right)}\right\}, q\right)$ for $L_{y}=\left(\right.$ a) 3 , (b) 4 , where $\left\{G_{t\left(L_{y}\right)}\right\}$ denotes the $\left(L_{x}=\infty \times L_{y}\right.$ strip of the triangular lattice. For comparison, the zeros of the chromatic polynomials $P\left(\left(G_{t\left(L_{y}\right)}\right)_{m}, q\right)$ for (a) $L_{y}=3, m=12$ (hence, $n=42$ ) and (b) $L_{y}=4, m=12$ (hence $n=56$ ) are shown.

In Figs. $5(a)$ and 5 (b) we show similar results for strips of width $L_{y}=3$ and $L_{y}=4$ of the triangular lattice. One sees a significant change in $\mathcal{B}$ as the width increases from 3 to 4 ; the self-conjugate arc crossing the real axis at $q=2.56984 \ldots$ for $L_{y}=3$ disappears, while what were previously the right-hand ends of the complex-conjugate arcs become multiple points of index 3, each sprouting two additional arcs pointing in toward the real axis. The patterns of chromatic zeros for the long finite strips also change in the same manner, so that, as expected, for each width, these zeros lie close to the respective asymptotic loci $\mathcal{B}$. Our explicit result for $W$ for the $L_{y}=3$ strip is

$$
\begin{aligned}
W\left(\left\{G_{t(3)}\right\}, q\right)= & 2^{-1 / 3}\left[q^{3}-7 q^{2}+18 q-17+\right. \\
& {\left.\left[q^{6}-14 q^{5}+81 q^{4}-250 q^{3}+442 q^{2}-436 q+193\right]^{1 / 2}\right]^{1 / 3} }
\end{aligned}
$$

for real $q$ to the right of the crossing at $q=2.56984$ noted above, with appropriate analytic continuation to the rest of the $q$ plane. 


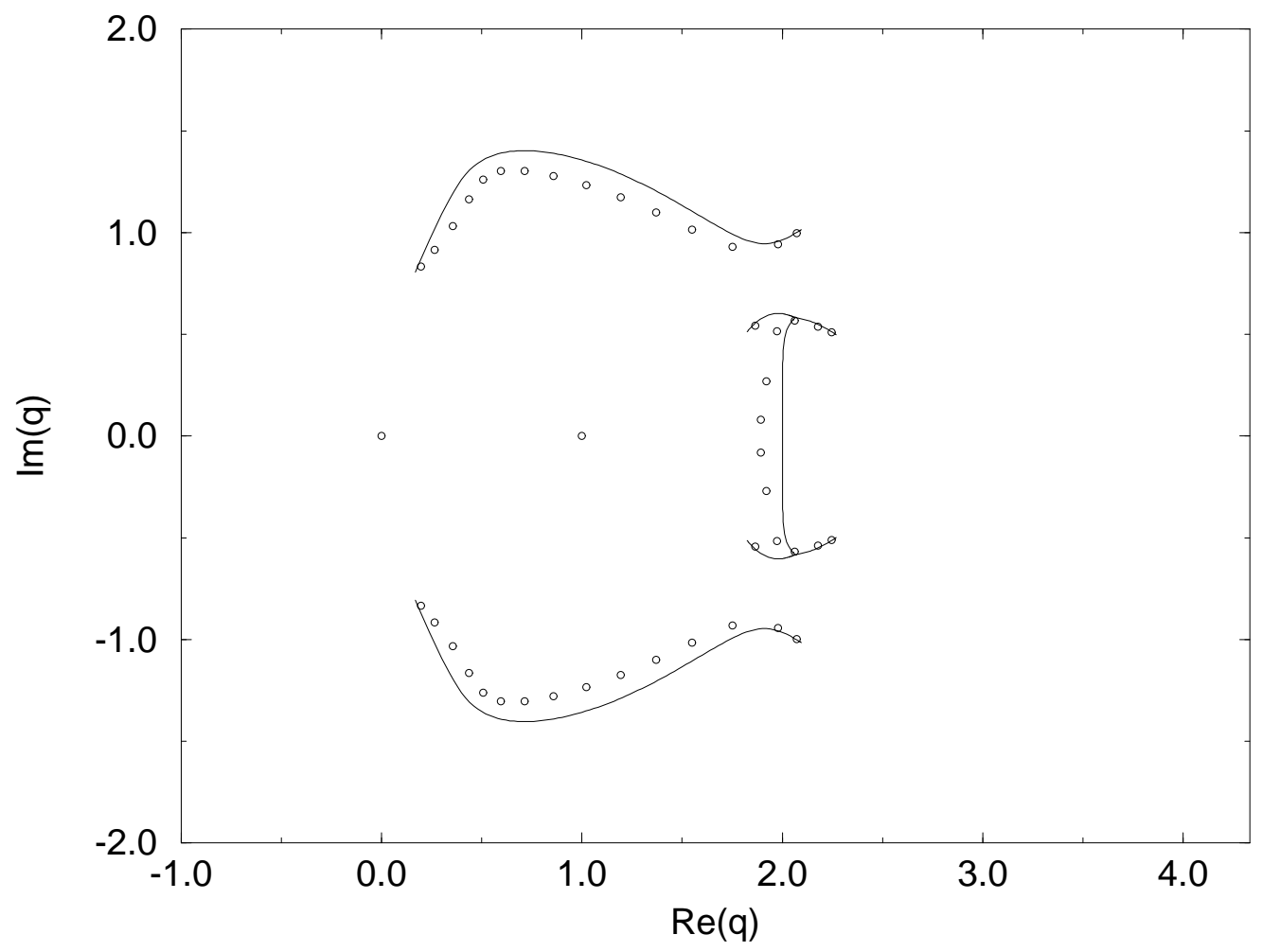

FIG. 6. Analytic structure of the function $W\left(\left\{G_{h c(3)}\right\}, q\right)$, where $\left\{G_{h c(3)}\right\}$ the $L_{x} \times L_{y}=\infty \times 3$ strip of the honeycomb (brick) lattice. Zeros of the chromatic polynomial $P\left(\left(G_{h c(3)}\right)_{6}, q\right)(n=46)$ are shown.

In Fig. 6 we show our calculations of $\mathcal{B}$ for a $L_{x} \times L_{y}=\infty \times 3$ strip of the honeycomb (brick) lattice. As was true with the square and triangular lattices, the arcs help one understand the pattern of chromatic zeros calculated for an $8 \times 8$ lattice with the same free boundary conditions in Ref. [20]; the positions of these arcs are close to the positions of the chromatic zeros, both for long finite strips and the $8 \times 8$ patch. Furthermore, the cusp-like structures in the latter case occur approximately where (i) our exact arcs have multiple points and (ii) there are gaps between the arcs.

Using our calculations of the generating functions, we have also determined the exact $W$ functions and their associated continuous loci of nonanalyticities $\mathcal{B}$ for infinitely long strips of three heteropolygonal lattices, viz., kagomé $(=(3 \cdot 6 \cdot 3 \cdot 6)),\left(3 \cdot 12^{2}\right)$, and $\left(4 \cdot 8^{2}\right)$, in the standard mathematical notation of eq. (3.17). As an example, we consider the kagomé lattice. In Fig. 7 we show our exact determination of $\mathcal{B}$ for an infinite strip 
of this type, a finite section of which was shown in Fig. 国(f). There is a particular feature of this calculation that is worth noting: for strip graphs which have $\mathcal{D}$ functions that are quadratic in $x$, the $\lambda_{s, k}(q)$ 's given by eq. (4.13) do not, in general, factorize. However, for the strip of the kagomé lattice considered here, the $\lambda_{s, k}(q)$ 's do factorize. This happens because $b_{k a g(2), 1}$ and the square root of the discriminant both have a common factor, $(q-2)$. Related to this, not all of the zeros of the discriminant are branch point singularities of the square root of this discriminant; the zero at $q=2$ is of even order (a double zero), and hence is not associated with a branch point of the square root of the discriminant (although it is a branch point singularity of the full $W$ function; see below). We calculate

$$
\begin{aligned}
W\left(\left\{G_{k a g(2)}\right\}, q\right)= & 2^{-1 / 5}(q-2)^{1 / 5}\left[q^{4}-6 q^{3}+14 q^{2}-16 q+10\right. \\
& \left.+\left[q^{8}-12 q^{7}+64 q^{6}-200 q^{5}+404 q^{4}-548 q^{3}+500 q^{2}-292 q+92\right]^{1 / 2}\right]^{1 / 5}
\end{aligned}
$$

As before, one can obtain this for positive real $q$ and analytically continue the expression to the full complex $q$ plane, except for the locus $\mathcal{B}$ where $W\left(\left\{G_{k a g(2)}\right\}, q\right)$ is nonanalytic. Note that although the point $q=2$ is a branch point singularity of $W\left(\left\{G_{k a g(2)}\right\}, q\right)$, it is an isolated singularity, and does not lie on the continuous locus of nonanalytic points $\mathcal{B}$. 


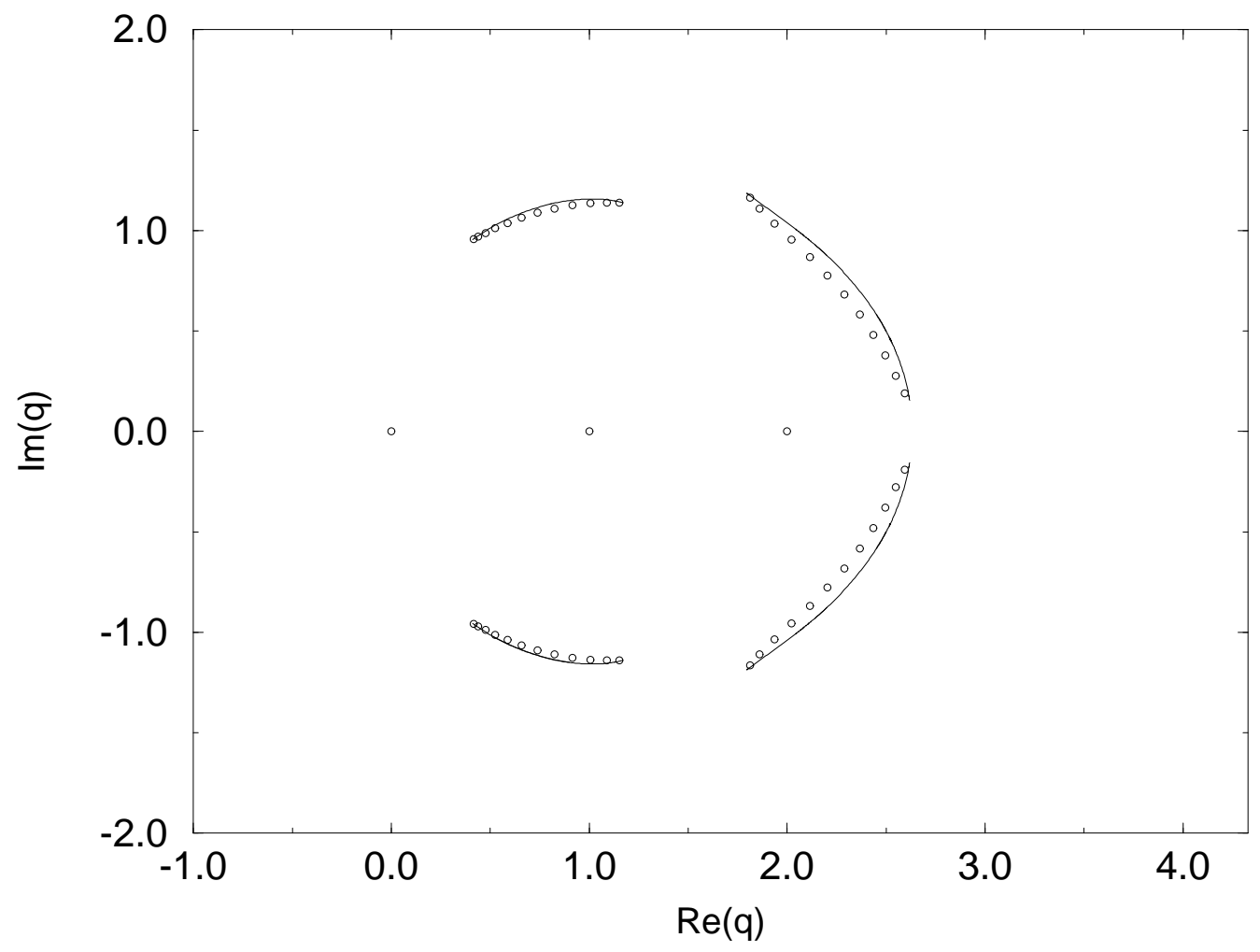

FIG. 7. Analytic structure of the function $W\left(\left\{G_{k q q(2)}\right\}, q\right)$, where $\left\{G_{k a g(2)}\right\}$ denotes an infinitely long strip of the kagomé lattice, a finite-length section of which is shown in Fig. 1 1 (f). Zeros of the chromatic polynomial $P\left(\left(G_{k a g(2)}\right) 9, q\right)$ of a finite strip with $m=9$ (hence $n=51$ ) are shown.

We present our results for the generating functions of the other heteropolygonal Archimedean lattices in Appendix 2 and the resultant determinations of $\mathcal{B}$ in the figures of Appendix 3. The chromatic zeros for long finite strips of each of these types are also shown in these figures. As with homopolygonal lattices, the arcs for the $w=1\left(3 \cdot 12^{2}\right)$ and $w=2\left(4 \cdot 8^{2}\right)$ infinite strips show close similarity in position not only with the chromatic zeros calculated for finite strips of the same type and width but also with our previous calculations of chromatic zeros for reasonably large patches of the respective lattices with $L_{x} \simeq L_{y}$ in Ref. [24]. For the $\left(3 \cdot 12^{2}\right)$ lattice, the large number (nine) of closely spaced arcs in Fig. 8 correlates nicely with the rather smooth and round distribution of chromatic zeros that we presented in Fig. 8 of Ref. [24]. For the $\left(4 \cdot 8^{2}\right)$ lattice, the correspondence with the chromatic zeros of Fig. 9 in Ref. [24] is close for the $w=2$ 
strip, shown in Fig. 95 here, as before, one sees a faithful correspondence between cusp-like structures in the chromatic zeros on the $2 \mathrm{D}$ path and the multiple points and gaps between arcs on $\mathcal{B}$ for the strip.

There are a number of interesting features exhibited by our results. A general feature is that for all of these strip graphs of the form $G_{s}=\left(\prod_{\ell=1}^{m} H\right) I$ as in eq. (1.2), the respective loci $\mathcal{B}$ form arcs which do not separate the complex $q$ plane into different, disconnected regions. This finding may be compared with our exact results in Refs. [21,23. There we showed that the nature of the locus $\mathcal{B}$ depends strongly on the topology of a graph, (boundary conditions for a lattice graph). In the simplest case of an $n$-vertex tree graph $T_{n}$, in the limit $n \rightarrow \infty, W(\{T\}, q)$ is analytic in the entire finite $q$ plane (i.e., $\mathcal{B}=\emptyset$, the null set), whereas if one ties together the ends to make an $n$-vertex circuit graph $C_{n}$, then in the limit $n \rightarrow \infty, W(\{C\}, q)$ is nonanalytic on the boundary $\mathcal{B}$ formed by the circle $|q-1|=1$, which divides the $q$ plane into two regions [21. Similarly, the $n \rightarrow \infty$ limit of an $n$-vertex strip graph (chain) composed of $m p$-sided polygons, denoted $(C h)_{p, n}$ in Ref. [23] (with $n=(p-2) m+2$ ) yields a $W\left(\left\{(C h)_{p}\right\}, q\right)$ function with $\mathcal{B}=\emptyset$, whereas if one ties together the ends to form a circular ladder graph, the resultant function $W(\{L\}, q)$ is nonanalytic on a continuous locus $\mathcal{B}$ which forms the boundaries of four different regions in the $q$ plane (as shown in Fig. 3 of Ref. 21]). In all of these cases, $\mathcal{B}$ was either (i) the null set $\emptyset$, for the $n \rightarrow \infty$ limit of $n$-vertex graphs such as the complete graph $K_{n}$, tree graph $T_{n}$, and strip (chain) graph composed of $p$-gons, $(C h)_{p, n}$, or (ii) a set of boundaries that separated the complex $q$ plane into two or more different regions, within which $W(\{G\}, q)$ was analytic, such as for the circuit, $C_{n}$, periodic and twisted ladder graphs, and others like the wheel graph and its generalization to the $p$-wheel graph 21,23]. Thus, the strip graphs studied here yield $W\left(\left\{G_{s}\right\}, q\right)$ functions with analytic structure intermediate between type (i) and (ii) found earlier: $\mathcal{B}$ is nonempty but does not divide the $q$ plane into separate regions of analyticity. Moreover, different strips exhibit a wide variety in the number of arcs. As we shall discuss in Ref. 29], we have found that for strip graphs of the form $J\left(\prod_{\ell=1}^{m} H\right) I, \mathcal{B}$ contains arcs again, but these can actually enclose regions; this happens if the degeneracy equation (4.16) involves at least one $\lambda_{s, k}$ which is a polynomial in $q$. In contrast, for all of the strip graphs of the form $\left(\prod_{\ell=1}^{m} H\right) I$ and having $\mathcal{B} \neq \emptyset$ considered in the present paper, the maximal $\lambda_{s, k}$ 's that occur in the degeneracy equation (4.16) are algebraic, but not simply polynomial, functions of $q$, i.e., they involve branch point singularities.

The origin of this arc structure of $\mathcal{B}$ for the strip graphs studied here can be seen explicitly by examining the calculation of this locus in the simple case where $k_{\text {max }}=2$, i.e., where the denominator $\mathcal{D}\left(G_{s}, q, x\right)$ of the generating function $\Gamma\left(G_{s}, q, x\right)$ is quadratic as a polynomial in $x$. Here, aside from the trivial case where for some $q, b_{s, 1}(q)=b_{s, 2}(q)=0$ 国 the continuous locus of points $\mathcal{B}$ where $W\left(\left\{G_{s}\right\}, q\right)$ is nonanalytic

\footnotetext{
7 The $n$-vertex wheel graph $(W h)_{n}$ is the graph formed by adjoining a point (i.e., connecting it with bonds) to all vertices of an $(n-1)$-vertex circuit graph $C_{n-1}$. This may be denoted $(W h)_{n}=K_{1} \times C_{n-1}$, where $K_{\ell}$ is the complete graph on $\ell$ vertices. The generalized $p$-wheel graph is given by $(W h)_{n}^{(p)}=K_{p} \times C_{n-p}$ with $(W h)_{n}^{(1)} \equiv(W h)_{n}[21] 23$.

${ }^{8}$ The restriction to nonvanishing $b_{s, k}$ is relevant for the $\mathcal{D}$ functions for the kagomé $($ i.e., $(3 \cdot 6 \cdot 3 \cdot 6))$ and $\left(3 \cdot 12^{2}\right)$
} 
is determined by the special case of eq. (4.16) with $\lambda_{s, k}(q), k=1,2$ given by eq. (4.13). The arcs comprising $\mathcal{B}$ extend between, and end on, zeros of the discriminant. It follows that $N_{\text {arc }}=N_{\text {b.p. }} / 2$, where $N_{\text {arc }}$ and $N_{b . p .}$ denote the number of arcs and branch points, respectively. In Table 1 we have listed these for the strip graphs with $k_{\max }=2$ considered here. An analogous connection holds between the arc endpoints and branch points in the maximal-magnitude $\lambda_{s, k}$ 's appearing in the degeneracy equation (4.16) for the case $k_{\max }=3$, and one can see that it is a general feature of the degeneracy equations for $\lambda_{s, k}$ 's of maximal magnitude. Because $\mathcal{B}$ is invariant under complex conjugation, it follows that the arcs must occur as complex-conjugate pairs together with possible self-conjugate arcs. Clearly if $N_{\text {arc }}$ is odd, then there must exist at least one such self-conjugate arc. One can see by scanning Table 1 and the associated figures various examples illustrating both the presence and absence of self-conjugate arcs.

The fact that $\mathcal{B}$ consists of arcs ending on branch points of the maximal-magnitude $\lambda_{s, k}$ in the complex $q$ plane is reminiscent of the appearance of arcs (and line segments) of nonanalyticities of the free energy, ending on branch points of maximal-magnitude eigenvalues of the transfer matrix, in exact calculations of complex-temperature phase diagrams of 1D systems (e.g., [33, 35, 36]) and finite-width strips [37]. Our earlier work on complex-temperature phase diagrams [33,35,36] helps to elucidate the conditions under which arcs or line segments do or do not enclose distinct regions in the respective complex plane (here, the $q$ plane, there the complex plane in an appropriate Boltzmann weight variable). For example, in the 1D nearest-neighbor Ising model with spin $s=1 / 2$, the eigenvalues of the transfer matrix are given by $\lambda_{1 / 2, \pm}=u^{-1 / 4}(1 \pm \sqrt{u})$, and hence $\mathcal{B}$ consists of the semi-infinite line segment $\infty \leq u \leq 0$, where $u=e^{-4 K}$ with $K=\beta J, \beta=1 /\left(k_{B} T\right)$, and $T$ and $J$ are the temperature and spin-spin coupling. This locus of points ends on the branch point $u=0$ and does not separate the complex $u$ plane into different regions. In contrast, for spin $s=1$, the eigenvalues of the transfer matrix are $\lambda_{1,1}=u_{1}^{-1}-u_{1}$ and

$$
\lambda_{1, \pm}=(1 / 2)\left[u_{1}^{-1}+1+u_{1} \pm\left(u_{1}^{-2}-2 u_{1}^{-1}+11-2 u_{1}+u_{1}^{2}\right)^{1 / 2}\right]
$$

where $u_{1}=e^{-K}$; here the curves comprising $\mathcal{B}$, which are the solutions to the degeneracy equation of maximal eigenvalues of the transfer matrix $\left|\lambda_{1,1}\right|=\left|\lambda_{1,+}\right|$, do separate the complex $u_{1}$ plane into (four) different regions, as shown in Fig. 1(a) of Ref. [35. These curves end on the singular point at $u_{1}=0$ and extend to complex infinity. Note that no arcs end on the finite branch points of the square root in eq. (5.8) because neither $\lambda_{1,+}$ nor $\lambda_{1,-}$ is a dominant eigenvalue at any of these points. Related to this, when one crosses one of the curves, one does not switch between two $\lambda$ 's that are related only by changing the sign of

strips of width $L_{y}=2$; since both of these strips contain triangles, the chromatic polynomials vanish for all $m \geq 1$ if $q=2$. This is reflected in the property that for $q=2$, the coefficient functions $a_{s, 1}=b_{s, 1}=b_{s, 2}=0$, and the respective generating functions become $x$-independent. Hence, as is evident from Figs. 7 and 8 , the point $q=2$ is not on the nonanalytic locus $\mathcal{B}$. Related to this, as we have indicated in Table 1 , in these two cases, although the

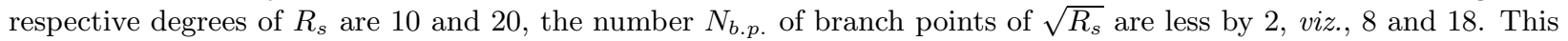
in contrast to the other strips listed in Table 1 , where $\operatorname{deg}\left(R_{s}\right)=N_{b . p}$. 
the square root, in contrast, e.g., to the situation described by eq. 4.16) for strip graphs considered here. One also encounters arcs which close in such a manner as to separate the complex-temperature plane into different regions which are compact, rather than extending to infinity. This occurs, for example, in the case of the $1 \mathrm{D} \mathbb{Z}_{6}$ clock model [33] and the $1 \mathrm{D}$ spin $1 / 2$ Ising model with nearest- and next-nearest-neighbor spin-spin couplings $J_{N N}$ and $J_{N N N}$ for certain values of the ratio $J_{N N N} / J_{N N}$ [36].

Another salient feature of our results is that although in some cases there is often a self-conjugate arc which crosses the real $q$ axis farthest to the right, such an arc does not occur farthest to the left. This is also reflected in the reduced density (along arcs) of chromatic zeros near to $q=0$ (aside from the zero precisely at $q=0$, which is always present for any chromatic polynomial).

Our current results are relevant to the question of the conditions under which components of $\mathcal{B}$ are compact or, in contrast, extend infinitely far from the origin of the $q$ plane, yielding a reduced function $\bar{W}(\{G\}, q)$ which is nonanalytic at $1 / q=0$. A family of graphs with noncompact $\mathcal{B}$ was studied in Refs. [28,21], and in Ref. 23] infinitely many families of graphs were constructed with noncompact curves $\mathcal{B}$, leading to the formulation of a general condition explaining when such noncompactness does or does not occur. Since for the $G_{s}$ strip graphs considered here the branch points of the maximum-magnitude $\lambda_{s, k}$ 's, and hence the endpoints of the arcs, occur at finite values of $q$, these arcs never extend infinitely far from the origin $q=0$. This is in accord with the criterion for compactness of $\mathcal{B}$ presented in our earlier general study [23].

A longstanding question in graph theory has concerned general features of chromatic zeros for a general graph $G$. Given the merging of a subset of these in the infinite-vertex limit to form the locus $\mathcal{B}$ of points where $W(\{G\}, q)$ is nonanalytic, one is also led to ask about general features of $\mathcal{B}$ for an arbitrary graph. We have discussed several of these above. Here we formulate a conjecture concerning the sign of the real part of chromatic zeros and thus of points on $\mathcal{B}$. Before doing this, we recall that some time ago, it was conjectured [38] that the chromatic zeros of an arbitrary graph have non-negative real parts. This conjecture was subsequently shown to be false, and now many examples of graphs are known with chromatic zeros having negative real parts. However, a striking feature of our exact calculations of chromatic zeros and nonanalytic loci $\mathcal{B}$ in Refs. [21,23, 24, for many families of graphs, as well as the calculations of chromatic zeros for lattices with free, as opposed to cylindrical, boundary conditions, in Ref. [20], is that they have $\operatorname{Re}(q) \geq 0$. We are thus led to state the following conjectures. We first give a definition: a regular lattice graph has what we denote as a "global circuit" if and only if by travelling along one of the lattice vectors one can arrive back at one's starting point. The definition of lattice vector is clear for a cartesian $d$-dimensional lattice; for the triangular and honeycomb lattices, the lattice vectors can be taken to lie along $0^{\circ}, 60^{\circ}$, and $120^{\circ}$, etc. Thus, a patch of any of these lattices with free boundary conditions has no global circuits, while a patch with periodic boundary conditions in at least one direction has global circuits along this direction.

\section{Conjecture 1a}

If $q_{0}$ is a chromatic zero of a regular lattice graph $G$ with no global circuits, then $\operatorname{Re}\left(q_{0}\right) \geq 0$. Furthermore, 
the only chromatic zero with $\operatorname{Re}\left(q_{0}\right)=0$ is $q_{0}=0$ itself.

Conjecture $1 b$

Consider the $n \rightarrow \infty$ limit of a regular lattice graph $G$ with no global circuits. The points $q \in \mathcal{B}$ have the property that $\operatorname{Re}(q) \geq 0$. Furthermore, the only point on $\mathcal{B}$ with $\operatorname{Re}(q)=0$ is the point $q=0$ itself.

Note that our exact calculations in Refs. 21,23 show that a graph may have global circuits and still have chromatic zeros and, in the infinite- $n$ limit, a locus $\mathcal{B}$, with the properties that (i) $\operatorname{Re}(q) \geq 0$ and (ii) $R e(q)=0 \Rightarrow q=0$; this was true, in particular, of the circuit graph, its generalization [23] to $K_{p} \times C_{n-p}$. This is also true of the ladder graph (strip of the square lattice of width $L_{y}=2$ with ends tied together (in an untwisted or twisted manner), i.e., with periodic boundary conditions in the longitudinal direction [21], both of which have global circuits along the longitudinal direction. This shows that the condition of no global circuits is a sufficient, but not necessary, condition for properties (i) and (ii) above. Although our previous (exact) calculations of chromatic polynomials and $W$ functions in Refs. [21,23] showed that these are affected by boundary conditions, it also showed that, in all the cases considered, when one compared a given type of graph with different topology (i.e., existence or nonexistence of global circuits) due to different boundary conditions, there did exist a unique value $q_{c}$ such that for real $q \geq q_{c}$, the $W$ functions calculated with the different boundary conditions coincided. For example, for the line graph with free boundary conditions (and more generally the tree graph), in the infinite-vertex limit, $W(\{T\}, q)=q-1$, while for the circuit graph $C_{n}$ (i.e. the line graph with periodic boundary conditions), in the same limit, $W(\{C\}, q)=q-1$ for $|q-1|>1$ and $|W(\{C\}, q)|=1$ for $|q-1|<1$ (see section IV of Ref. [21]). Thus, $W(\{T\}, q)=W(\{C\}, q)$ for real $q \geq q_{c}$, where $q_{c}=2$ in this case (more generally, they also coincide throughout the region $|q-1| \geq 1$ in the complex $q$ plane). Similarly, for the $n$-vertex ladder graphs $L_{n}$, in the $n \rightarrow \infty$ limit with free boundary conditions, $W\left(\{L\}_{f b c}, q\right)=\left(q^{2}-3 q+3\right)^{1 / 2}$, while for (twisted or untwisted) periodic boundary conditions, this expression again holds in the region denoted $R_{1}$ in Ref. [21], defined in general as the maximal region which is analytically connected with the real interval $q \geq q_{c}$ for a given lattice?. For the periodic ladder graphs, $q_{c}$ is again equal to $2\left(R_{1}\right.$ also includes the interval $q<0$ on the real axis), and there are three other regions in the complex $q$ plane where $W$ takes on other analytic forms. These exact results suggest the following generalization: for a given type of strip graph $G_{s}$ of a regular lattice, for real $q \geq q_{c}$, where $q_{c}$ is the maximal (finite) real value at which $W\left(\left\{G_{s}\right\}, q\right)$ is nonanalytic (equivalently, $\mathcal{B}$ crosses the real axis) for some set of boundary conditions, the functions $W\left(\left\{G_{s}\right\}, q\right)$ calculated with the different sets of boundary conditions coincide. A further generalization is that these $W$ functions can be stated as follows. Consider the set of (in general different) loci $\mathcal{B}$ calculated for the infinite-vertex limit of a regular lattice with different

\footnotetext{
${ }^{9}$ In eqs. (4.22)-(4.24) of Ref. [21], the $W$ function was defined per rung rather than per site; for the definition per site, as used here, the right-hand sides of these equations are raised to the power $1 / 2$.
} 
boundary conditions. Consider the maximal envelope of $\mathcal{B}$ for this set. Then the $W$ functions agree in the region outside this maximal envelope, which region includes the area extending infinitely far away from the origin of the $q$ plane, i.e., the inverse image of the origin of the $1 / q$ plane. Note that this property is necessary for the existence of large- $q$ Taylor series expansions of $\bar{W}$ for regular lattices. As discussed in Refs. [21,23], the existence of these large- $q$ series expansions for $\bar{W}$ also depends on the property that in these cases $\mathcal{B}$ does not extend infinitely far away from the origin in the $q$ plane.

\section{CONCLUSIONS}

In conclusion, in this paper we have calculated the chromatic polynomials $P\left(G_{s}, q\right)$ for strip graphs comprised of repeated subgraphs $H$ adjoined to an initial graph $I$ and, from these, in the limit of infinitely long strips, the resultant $W\left(\left\{G_{s}\right\}, q\right)$ functions. To do this, we have developed a powerful method in which we compute a generating function $\Gamma\left(G_{s}, q, x\right)$ which yields these chromatic polynomials as coefficients in a series expansion in $x$. We have studied the analytic structure of $W\left(\left\{G_{s}\right\}, q\right)$ for a number of different types and widths of strip graphs. The locus of points where $W\left(\left\{G_{s}\right\}, q\right)$ is nonanalytic consists of arcs which do not separate the complex $q$ plane into different regions. From comparisons of arcs for different-width strips of given types, we have shown how these arcs elongate and tend to move toward each other as the width of the infinitely long strips is increased. We have calculated chromatic zeros for long finite-length strips and have shown that, aside from the generally occuring real zeros at $q=0,1$ and, for strips containing triangles, $q=2$, these lie close to the positions of the arcs. As we have discussed, these calculations of the ground state degeneracy $W(\Lambda, q)$ of the $q$-state Potts antiferromagnet on these infinitely long strips of various widths helps one to understand the behavior of this function on infinite $2 \mathrm{D}$ lattices $\Lambda$. In turn, this adds to our understanding of ground state degeneracy and entropy, a phenomenon which is exhibited in the physical world by such compounds as $\left(\mathrm{H}_{2} \mathrm{O}\right)$ ice.

\section{Acknowledgments}

This research was supported in part by the NSF grant PHY-93-09888.

\section{APPENDIX 1}

In this Appendix we present two alternate ways to calculate the generating function $\Gamma\left(G_{s}, q, x\right)$ that are complementary to the method given in section IIB. 


\section{A. Calculation of the Generating Function by Matching of Terms}

Here, as before, we solve for $\Gamma\left(G_{s}, q, x\right)$ simply by matching the Taylor series expansion of $\Gamma\left(G_{s}, q, x\right)$ to explicit calculations of the chromatic polynomials for the first few values of $m$. Since there are $N_{p}=$ $j_{\max }+k_{\max }+1$ polynomials in (2.10)-(2.12), we can determine these by (i) calculating the chromatic polynomials $P\left(\left(G_{s}\right)_{m}, q\right)$ for the various strips of type $G_{s}$ with $m$ ranging from 0 to $N_{p}$, (ii) performing the Taylor series expansion of $\Gamma\left(\left\{G_{s}\right\}, q, x\right)$ about $x=0$ to order $N_{p}-1$, (iii) equating the successive terms in the Taylor series expansion to the respective chromatic polynomials, using (2.9), and (iv) solving for the $a_{j}$, $j=0 . ., j_{\max }$ and $b_{k}, k=1 . ., k_{\max }$. For example, for many strip graphs of width $w=L_{y}-1=2$, we find that $j_{\max }=1, k_{\max }=2$ 19, so that $\Gamma\left(G_{s}, q, x\right)$ has the form (2.39) given above. Evaluating eq. (2.9), one has

$$
a_{s, 0}=P\left(\left(G_{s}\right)_{0}, q\right)
$$

(where $P\left(\left(G_{s}\right)_{0}, q\right)=P(I, q)$ from eq. 2.44))

$$
\begin{gathered}
a_{s, 1}-a_{s, 0} b_{s, 1}=P\left(\left(G_{s}\right)_{1}, q\right) \\
a_{s, 0} b_{s, 1}^{2}-a_{s, 0} b_{s, 2}-a_{s, 1} b_{s, 1}=P\left(\left(G_{s}\right)_{2}, q\right)
\end{gathered}
$$

and

$$
2 a_{s, 0} b_{s, 1} b_{s, 2}-a_{s, 1} b_{s, 2}+a_{s, 1} b_{s, 1}^{2}-a_{s, 0} b_{s, 1}^{3}=P\left(\left(G_{s}\right)_{3}, q\right)
$$

which has the solution given by (7.1) together with

$$
\begin{gathered}
a_{s, 1}=\frac{2 P\left(\left(G_{s}\right)_{0}, q\right) P\left(\left(G_{s}\right)_{1}, q\right) P\left(\left(G_{s}\right)_{2}, q\right)-P\left(\left(G_{s}\right)_{0}, q\right)^{2} P\left(\left(G_{s}\right)_{3}, q\right)-P\left(\left(G_{s}\right)_{1}, q\right)^{3}}{Q} \\
b_{s, 1}=\frac{P\left(\left(G_{s}\right)_{1}, q\right) P\left(\left(G_{s}\right)_{2}, q\right)-P\left(\left(G_{s}\right)_{0}, q\right) P\left(\left(G_{s}\right)_{3}, q\right)}{Q} \\
b_{s, 2}=\frac{P\left(\left(G_{s}\right)_{1}, q\right) P\left(\left(G_{s}\right)_{3}, q\right)-P\left(\left(G_{s}\right)_{2}, q\right)^{2}}{Q}
\end{gathered}
$$

where

$$
Q=P\left(\left(G_{s}\right)_{0}, q\right) P\left(\left(G_{s}\right)_{2}, q\right)-P\left(\left(G_{s}\right)_{1}, q\right)^{2}
$$

The resulting $a_{1}, b_{1}$, and $b_{2}$ are polynomials, although this is not manifest in eqs. (7.5)-(7.7).

\footnotetext{
10 The honeycomb strip graph is an exception; for $w=2$, the resultant generating function has $j_{\max }=2, k_{\max }=3$.
} 


\section{B. Calculation of the Generating Function by Addition-Contraction Theorem}

Another alternate method of calculation is based on the addition-contraction theorem from graph theory. This method is quite powerful and allows us also to calculate chromatic polynomials and asymptotic limiting functions $W$ for strip graphs with specific subgraphs at both ends, not just one. We first recall the statement of the addition-contraction theorem: let $G$ be a graph, and let $v$ and $v^{\prime}$ be two non-adjacent vertices in $G$. Form (i) the graph $G_{a d d}$. in which one adds a bond connecting $v$ and $v^{\prime}$, and (ii) the graph $G_{c o n t r}$ in which one identifies $v$ and $v^{\prime}$. Then the chromatic polynomial for $G$ is equal to the sum of the chromatic polynomial for the graphs $G_{a d d}$. and $G_{\text {contr. }}$. For our proof, with no loss of generality, it is convenient to take the initial subgraph $I$ in eq. (1.2) to be identical to the repeating subgraph unit $H$; once having calculated the generating function for this case, it is straightforward to obtain the generating function for the strip with an initial subgraph $I$ attached to the right end. By applying the addition-contraction theorem to the right-hand side of the initial $H$ subgraph in $\left(G_{s}^{\prime}\right)_{m}$, we obtain a set of strip graphs $\left(G_{s, j}^{\prime}\right)_{m}$ with complete subgraphs labeled by $j$ on the right-hand end. For example, let us consider a strip $\left(G_{s}^{\prime}\right)_{m}=\prod_{\ell=1}^{m} H$ with $L_{y}=4$ vertices in the transverse direction, and label the vertices on the right-hand end, in sequence, as $v_{1}, v_{2}, v_{3}, v_{4}$. Now apply the addition-contraction theorem to the pair $v_{1}, v_{4}$; this yields the equation

$$
P\left(\left(G_{s}^{\prime}\right)_{m}, q\right)=P\left(\left(G_{s, b(1,4)}^{\prime}\right)_{m}, q\right)+P\left(\left(G_{s, v_{1}=v_{4}}^{\prime}\right)_{m}, q\right)
$$

where $\left(G_{s, b(1,4)}^{\prime}\right)_{m}$ denotes the strip graph with the right-hand end modified by the addition of the bond connecting vertices $v_{1}$ and $v_{4}$, and $\left(G_{s, v_{1}=v_{4}}^{\prime}\right)_{m}$ denotes the strip graph with the right-end modified by identifying vertices $v_{1}$ and $v_{4}$. Applying the theorem again to each of these two strip graphs, one obtains the equation

$$
\begin{aligned}
P\left(\left(G_{s}^{\prime}\right)_{m}, q\right)= & P\left(\left(G_{s, K_{4}}^{\prime}\right)_{m}, q\right)+P\left(\left(G_{s, K_{3}\left(v_{1}=v_{4}\right)}^{\prime}\right)_{m}, q\right)+P\left(\left(G_{s, K_{3}\left(v_{1}=v_{3}\right)}^{\prime}\right)_{m}, q\right) \\
& +P\left(\left(G_{s, K_{3}\left(v_{2}=v_{4}\right)}^{\prime}\right)_{m}, q\right)+P\left(\left(G_{s, K_{2}\left(v_{1}=v_{3}, v_{2}=v_{4}\right)}^{\prime}\right)_{m}, q\right)
\end{aligned}
$$

Let us label the five resultant complete graphs on the right-hand end via the label $i$, with chromatic polynomials denoted by $P\left(\left(G_{s, i}^{\prime}\right)_{0}, q\right)$. Next, working one's way leftward from the right-hand end, apply the addition-contraction theorem in a similar manner to the next set of transverse vertices, and label the five resultant complete graphs with the label $j$. Define a (square) matrix $M$ (in this case $5 \times 5$ ) with elements consisting of the chromatic polynomials of this set of graphs with complete subgraphs $i$ on the right and $j$ on the left. This procedure transforms the initial strip into a sum of factorized strips with parts that overlap in complete graphs. Therefore the intersection theorem yields, for $m \geq 1$,

$$
P\left(\left(G_{s, j}^{\prime}\right)_{m}, q\right)=\sum_{i} \frac{M_{j i} P\left(\left(G_{s, i}^{\prime}\right)_{m-1}, q\right)}{P\left(\left(G_{s, i}^{\prime}\right)_{0}, q\right)}=\sum_{i}(M D)_{j i} P\left(\left(G_{s, i}^{\prime}\right)_{m-1}, q\right)
$$

where $D$ is a $(5 \times 5)$ diagonal matrix with elements $D_{i, i}=1 / P\left(\left(G_{s, i}^{\prime}\right)_{0}, q\right)$. Because of $(7.10)$, the generating function of $G_{s}^{\prime}$ can be written as 


$$
\Gamma\left(G_{s}^{\prime}, q, x\right)=\sum_{j} \Gamma\left(G_{s, j}^{\prime}, q, x\right)
$$

Writing the generating function of $G_{s, j}^{\prime}$ in the more convenient form

$$
\Gamma\left(G_{s, j}^{\prime}, q, x\right)=\sum_{m=1}^{\infty} P\left(\left(G_{s, j}^{\prime}\right)_{m}, q\right) x^{m-1}
$$

and using (7.11) and (7.12), we obtain

$$
\Gamma\left(G_{s}^{\prime}, q, x\right)=\mathbf{v}_{\mathbf{a}}(1-x M D)^{-1} M \mathbf{v}_{\mathbf{b}}=\mathbf{v}_{\mathbf{a}} \sum_{m=0}^{\infty} x^{m}(M D)^{m} M \mathbf{v}_{\mathbf{b}}
$$

where in this case, for the strip $G_{s}^{\prime}, \mathbf{v}_{\mathbf{a}}=(1,1,1,1,1)$ and $\mathbf{v}_{\mathbf{b}}=\mathbf{v}_{\mathbf{a}}{ }^{t}$ denotes the transpose of $\mathbf{v}_{\mathbf{a}}$. Note that in general $\mathbf{v}_{\mathbf{a}}$ and $\mathbf{v}_{\mathbf{b}}$ can be chosen independently, and different choices of these vectors correspond to different boundary conditions on the two ends of the strip. This approach to the theorem allows one to write (2.9) in the equivalent form

$$
P\left(\left(G_{s}^{\prime}\right)_{m}, q\right)=\mathbf{v}_{\mathbf{a}}(M D)^{m} M \mathbf{v}_{\mathbf{b}}
$$

The generating function (7.14) is a rational function of the form (2.10) with the denominator given by

$$
\mathcal{D}\left(G_{s}^{\prime}, q, x\right)=\prod_{r}\left(1-\lambda_{r}(q) x\right)
$$

where $\lambda_{r}(q)^{\prime} s$ are eigenvalues of the product of matrices $M D$. The boundary conditions on the two ends of the strip (defined by $\mathbf{v}_{\mathbf{a}}$ and $\mathbf{v}_{\mathbf{b}}$ ) determine which eigenvalues enter in the product in equation (7.16). Using these methods, we have also calculated chromatic polynomials and their asymptotic limits for various strip graphs of the form $\left(G_{s}\right)_{m}=J\left(\prod_{\ell=1}^{m} H\right) I$ (with $\left(G_{s}\right)_{0} \equiv J I$ ) where the strip has two, in general different, subgraphs on its ends. We shall present these calculations and related discussions of boundary conditions other than free ones in a subsequent paper [29].

\section{APPENDIX 2}

In this Appendix we list the $a_{s, j}$ and $b_{s, k}$ polynomials making up the generating functions $\Gamma\left(G_{s}, q, x\right)$ for various strip graphs $G_{s}$.

\section{A. Strip of the Square Lattice in the Diagonal Direction}

Here we consider a strip of the square lattice such that the longitudinal axis of the strip is the diagonal direction of the lattice. In the simplest case, where adjacent squares touch each other only at a common vertex, the chromatic polynomial factorizes, as $q(q-1) D_{4}(q)^{m+1}$ for $m$ squares adjoined to an initial one, $I$. The first case with a nonfactorizing chromatic polynomial is for the strip shown in Fig. 11(c), where the initial 
subgraph $I$ at the right-hand end is a single diagonally oriented square, and the repeating subgraph unit $H$ is a "wedge" consisting of two squares, one above the other, with a third to the immediate left. We denote the strip graph with $m$ of these repeating units $\left(G_{s q_{d}(3)}\right)_{m}$. We find a generating function with $j_{\max }=1$, $k_{\max }=2$, and

$$
\begin{gathered}
a_{s q_{d}(3), 0}=q(q-1) D_{4}(q) \\
a_{s q_{d}(3), 1}=-q(q-1)^{3}(q-2) \\
b_{s q_{d}(3), 1}=-q^{5}+8 q^{4}-28 q^{3}+53 q^{2}-56 q+27 \\
b_{s q_{d}(3), 2}=(q-1)(q-2) D_{4}(q)^{2}
\end{gathered}
$$

We have also calculated the generating function for a similar lattice of greater width, shown in Fig. 1 1 (d). For this case we find $j_{\max }=2, k_{\max }=3$, and

$$
\begin{gathered}
a_{s q_{d}(4), 0}=q(q-1) D_{4}(q)^{3} \\
a_{s q_{d}(4), 1}=-q(q-1)^{2} D_{4}(q)\left(3 q^{5}-25 q^{4}+86 q^{3}-152 q^{2}+136 q-47\right) \\
a_{s q_{d}(4), 2}=q(q-1)^{3}(q-2)^{2} D_{4}(q)^{3} \\
b_{s q_{d}(4), 1}=-q^{6}+10 q^{5}-45 q^{4}+116 q^{3}-185 q^{2}+178 q-82 \\
b_{s q_{d}(4), 2}=D_{4}(q)^{2}\left(3 q^{4}-22 q^{3}+64 q^{2}-88 q+49\right) \\
b_{s q_{d}(4), 3}=-(q-2)^{2} D_{4}(q)^{4}
\end{gathered}
$$

\section{B. Strip of the Triangular Lattice of Width $L_{y}=4$}

In the main text, we have given our results for the generating function for a strip of the triangular lattice with width $L_{y}=3$; here we list the results for the next wider strip of this type, having a width $L_{y}=4$. We calculate that $j_{\max }=3, k_{\max }=4$, and

$$
\begin{gathered}
a_{t(4), 0}=q(q-1)(q-2)^{6} \\
a_{t(4), 1}=-q(q-1)(q-2)^{3}(q-3)\left(3 q^{4}-25 q^{3}+76 q^{2}-98 q+43\right)
\end{gathered}
$$




$$
\begin{gathered}
a_{t(4), 2}=q(q-1)(q-2)^{6}(q-3)^{2}\left(3 q^{2}-12 q+8\right) \\
a_{t(4), 3}=-q(q-1)^{3}(q-2)^{6}(q-3)^{4} \\
b_{t(4), 1}=-q^{4}+10 q^{3}-42 q^{2}+88 q-76 \\
b_{t(4), 2}=(q-2)(q-3)^{2}\left(3 q^{3}-22 q^{2}+60 q-60\right) \\
b_{t(4), 3}=-(q-2)^{2}(q-3)^{3}\left(3 q^{3}-21 q^{2}+51 q-43\right) \\
b_{t(4), 4}=(q-2)^{6}(q-3)^{4}
\end{gathered}
$$

\section{Strip of the $\left(3 \cdot 12^{2}\right)$ Lattice}

We consider a strip oriented such that the upper and lower sides of the 12-gons are parallel to the direction of the strip. The width of the strip is given by the diameter of one 12-gon and the initial subgraph $I$ on the right is taken to be a 12-gon and the repeating subgraph unit $H$ is a 12-gon with its two adjacent triangles (which do not touch each other). We calculate $j_{\max }=1, k_{\max }=2$ and

$$
\begin{gathered}
a_{312(2), 0}=q(q-1) D_{12}(q) \\
a_{312(2), 1}=-q(q-1)^{10}(q-2)\left(q^{2}-5 q+5\right) \\
b_{312(2), 1}=-(q-2)\left(q^{9}-11 q^{8}+54 q^{7}-156 q^{6}+294 q^{5}-378 q^{4}+336 q^{3}-204 q^{2}+82 q-22\right) \\
b_{312(2), 2}=(q-1)^{7}(q-2)^{3}\left(q^{2}-5 q+5\right)
\end{gathered}
$$

\section{Strip of the $\left(4 \cdot 8^{2}\right)$ Lattice}

The minimal width strip of the $\left(4 \cdot 8^{2}\right)$ lattice is shown in Fig. 1 $1(\mathrm{~g})$. The initial subgraph $I$ is an octagon and the repeating subgraph unit $H$ is an octagon with two squares at the same $x$ coordinate (which do not touch each other). For this strip we find $j_{\max }=1$ and $k_{\max }=2$ and calculate

$$
\begin{gathered}
a_{488(2), 0}=q(q-1) D_{8}(q) \\
a_{488(2), 1}=-q(q-1)^{6}(q-2)\left(3 q^{2}-9 q+7\right)
\end{gathered}
$$




$$
\begin{gathered}
b_{488(2), 1}=-q^{8}+11 q^{7}-55 q^{6}+163 q^{5}-314 q^{4}+406 q^{3}-354 q^{2}+203 q-63 \\
b_{488(2), 2}=(q-1)^{3}(q-2)\left(3 q^{2}-9 q+7\right) D_{4}(q)^{2}
\end{gathered}
$$

We have also calculated the generating function for the next wider strip of the $\left(4 \cdot 8^{2}\right)$ lattice, shown in Fig. If(h). The longitudinal direction of this strip is rotated $45^{\circ}$ relative to that of the one above. We find $j_{\max }=2, k_{\max }=3$, and calculate

$$
\begin{gathered}
a_{488(3), 0}=q(q-1)\left(q^{14}-18 q^{13}+153 q^{12}-814 q^{11}+3030 q^{10}-8358 q^{9}+17655 q^{8}-29104 q^{7}\right. \\
\left.+37796 q^{6}-38720 q^{5}+31057 q^{4}-19118 q^{3}+8666 q^{2}-2649 q+427\right) \\
a_{488(3), 1}=-q(q-1)^{4}\left(q^{15}-23 q^{14}+251 q^{13}-1719 q^{12}+8241 q^{11}-29235 q^{10}\right. \\
+79172 q^{9}-166558 q^{8}+274466 q^{7}-354610 q^{6}+356940 q^{5} \\
\left.-275630 q^{4}+158575 q^{3}-64360 q^{2}+16492 q-1999\right) \\
a_{488(3), 2}=q(q-1)^{11}(q-2)^{2}(q-3)^{2} D_{4}(q)^{2} \\
b_{488(3), 1}=-q^{12}+16 q^{11}-120 q^{10}+558 q^{9}-1794 q^{8}+4212 q^{7}-7437 q^{6} \\
+10018 q^{5}-10324 q^{4}+8064 q^{3}-4648 q^{2}+1854 q-414 \\
b_{488(3), 2}=(q-1)^{4}\left(q^{12}-20 q^{11}+188 q^{10}-1094 q^{9}+4375 q^{8}-12640 q^{7}+27033 q^{6}\right. \\
\left.-43164 q^{5}+51235 q^{4}-44380 q^{3}+26931 q^{2}-10462 q+2017\right) \\
b_{488(3), 3}=-(q-1)^{8}(q-2)^{2}(q-3)^{2} D_{4}(q)^{2}
\end{gathered}
$$

\section{APPENDIX 3}

In this Appendix we include a number of additional figures depicting exact results on the analytic structure for $W\left(\left\{G_{s}\right\}, q\right)$ functions for heteropolygonal Archimedean lattices $G_{s}$, together with calculations of chromatic zeros for long finite strips of each type. 


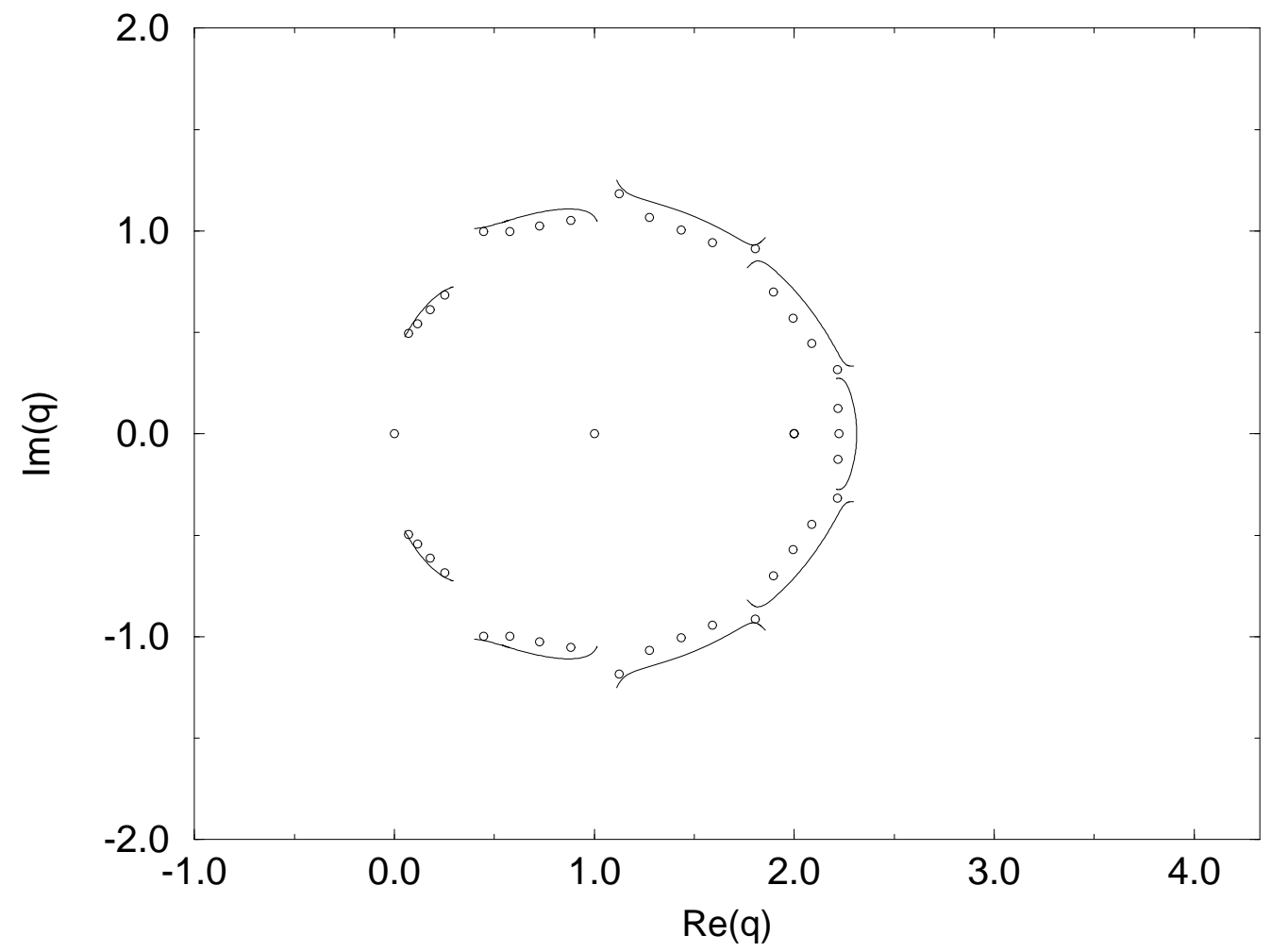

FIG. 8. Analytic structure of the function $W\left(\left\{G_{\left(3 \cdot 12^{2}\right)}\right\}, q\right)$, where $\left\{G_{\left.3 \cdot 12^{2}\right)}\right\}$ denotes an infinitely long strip of the $\left(3 \cdot 12^{2}\right)$ lattice oriented as discussed in the text, and of width one layer of 12-gons. For comparison, the zeros of the chromatic polynomial $\left.P\left(G_{\left(3 \cdot 12^{2}\right)}\right)_{3}, q\right)$ of a finite strip with $m=3$ (hence $n=42$ vertices), are shown. 


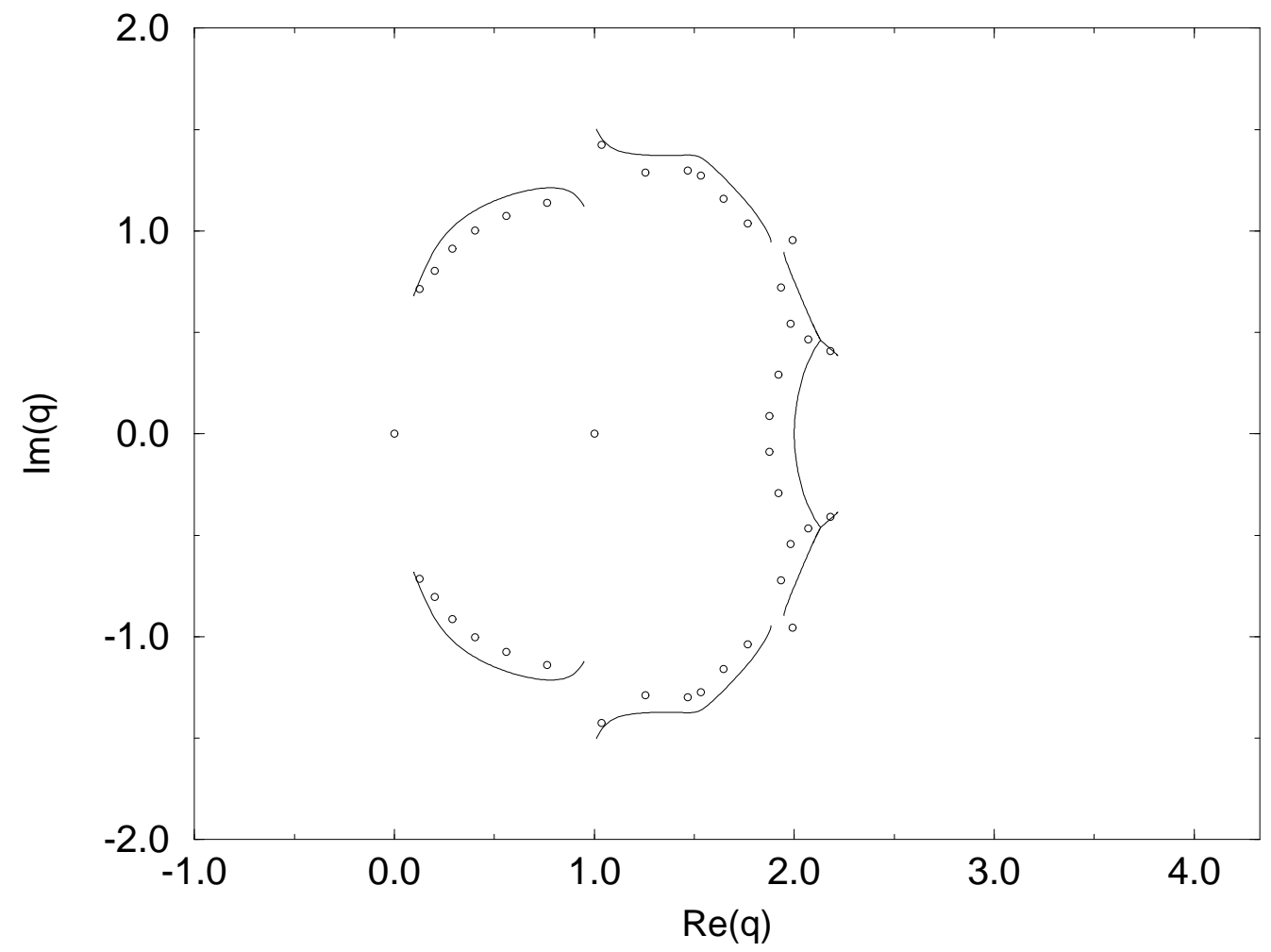

FIG. 9. Analytic structure of the function $W\left(\left\{G_{\left(4 \cdot 8^{2}\right)(2)}\right\}, q\right)$, where $\left\{G_{\left(4 \cdot 8^{2}\right)(w)}\right\}$ denotes an infinitely long strip of the $\left(4 \cdot 8^{2}\right)$ lattice of width $w$; a finite-length section is shown in Fig. $\mathbb{A}(\mathrm{h})$. For comparison, the zeros of the chromatic polynomial $P\left(\left(G_{\left(4 \cdot 8^{2}\right)(3)}\right)_{2}, q\right)$ of a finite strip (with $n=40$ ) is shown. 
[1] W. F. Giauque and J. W. Stout, J. Am. Chem. Soc. 58 (1936) 1144).

[2] L. Pauling, The Nature of the Chemical Bond (Cornell Univ. Press, Ithaca, 1960), p. 466.

[3] E. H. Lieb and F. Y. Wu, in C. Domb and M. S. Green, eds., Phase Transitions and Critical Phenomena (Academic Press, New York, 1972) v. 1, p. 331.

[4] N. G. Parsonage and L. A. K. Staveley, Disorder in Crystals (Oxford, 1978).

[5] P. W. Atkins, Physical Chemistry (Freeman, San Francisco, 1994), p. 710.

[6] G. H. Wannier, Phys. Rev. 79 (1950) 357.

[7] K. Kano and S. Naya, Prog. Theor. Phys. 10 (1953) 158.

[8] A. P. Ramirez, G. P. Espinosa, and A. S. Cooper, Phys. Rev. Lett. 64 (1990) 2070; C. Broholm, G. Aeppli, G. P. Espinosa, and A. S. Cooper, ibid. 65 (1990) 3173.

[9] A. S. Wills, A. Harrison, S. A. M. Mentink, T. E. Mason, and Z. Tun, "Magnetic Correlations in Deuteronium Jarosite, a Model $S=5 / 2$ Kagomé Antiferromagnet", cond-mat/9607106.

[10] C. Zeng and V. Elser, Phys. Rev. B42 (1990) 8436; R. R. P. Singh and D. Huse, Phys. Rev. Lett. 68 (1992) 1766; N. Elstner, R. R. P. Singh, and A. P. Young, ibid 71 (1993) 1629; N. Elstner and A. P. Young, Phys. Rev. B50 (1994) 6871.

[11] R. B. Potts, Proc. Camb. Phil. Soc. 48 (1952) 106.

[12] F. Y. Wu, Rev. Mod. Phys. 54 (1982) 235.

[13] G. D. Birkhoff, Ann. of Math. 14 (1912) 42.

[14] H. Whitney, Ann. of Math. 33 (1932) 688; Bull. Am. Math. Soc. 38 (1932) 572.

[15] G. D. Birkhoff and D. C. Lewis, Trans. Am. Math. Soc. 60 (1946) 355.

[16] R. C. Read, J. Combin. Theory 4 (1968) 52; R. C. Read and W. T. Tutte, "Chromatic Polynomials", in Selected Topics in Graph Theory, 3, eds. L. W. Beineke and R. J. Wilson (Academic Press, New York, 1988; R. Nelson and R. J. Wilson, eds., Graph Colourings (Longman, Essex, 1990).

[17] W. T. Tutte Graph Theory, vol. 21 of Encyclopedia of Mathematics and its Applications, ed. Rota, G. C. (AddisonWesley, New York, 1984).

[18] F. Harary, Graph Theory (Addison-Wesley, Reading, 1969); N. L. Biggs, Algebraic Graph Theory, (Cambridge, U.K., Cambridge Univ. Press, 1st ed. 1974, 2nd ed. 1993).

[19] E. H. Lieb, Phys. Rev. 162 (1967) 162.

[20] R. J. Baxter, J. Phys. A 20 (1987) 5241. See also R. J. Baxter, J. Phys. A 19 (1986) 2821.

[21] R. Shrock and S.-H. Tsai, Phys. Rev. E55 (1997) 5165.

[22] R. Shrock and S.-H. Tsai, J. Phys. A 30 (1997) 495; Phys. Rev. E55 (1997) 6791; Phys. Rev. E56 (1997) 2733.

[23] R. Shrock and S.-H. Tsai, Phys. Rev. E56 (1997) 1342; Phys. Rev. E56 (1997) 3935.

[24] R. Shrock and S.-H. Tsai, Phys. Rev. E56 (1997) 4111.

[25] J. Salas and A. Sokal, J. Stat. Phys. 86 (1997) 551.

[26] N. L. Biggs, R. M. Damerell, and D. A. Sands, J. Combin. Theory B 12 (1972) 123; N. L. Biggs and G. H. J. Meredith, J. Combin. Theory B 20 (1976) 5. The region diagram for the infinite- $n$ limit of ladder graphs, shown as Fig. 1 of the first paper, was not completely correct; the right-most complex-conjugate curves of the boundary $\mathcal{B}$ that were thought to terminate in region $R_{1}$ actually do not, but instead continue all the way in to the real axis, meeting at $q=2$ and thus enclosing two further regions. See Ref. [28] and Fig. 3 of Ref. 21].

[27] S. Beraha, J. Kahane, and N. J. Weiss, J. Comb. Theory B 28 (1980) 52.

[28] R. C. Read and G. F. Royle, in Graph Theory, Combinatorics, and Applications (New York, Wiley, 1991), vol. 2, p. 1009; R. C. Read, in the Proceedings of the Fifth Caribbean Conference on Combinatorics and Computing, Barbados, 1988. The region diagram for the infinite- $n$ limit of ladder graphs, shown as Fig. 4 and 13 in these respective papers, was not completely correct; on the left, the complex-conjugate curves of the boundary $\mathcal{B}$ that were thought to terminate on the unit circle at $q \simeq 0.191 \pm 0.982 i$ (i.e., at $q=e^{ \pm i \theta_{e}}$ with $\theta_{e}=\arccos ((3-\sqrt{5}) / 4)$ ) actually do not, but instead continue all the way to the real axis, meeting at the origin, $q=0$, and thus enclosing another region, as shown in Fig. 3 of Ref. 21]. Professor Read has kindly informed us (private communication) that he has calculated recursion relations and chromatic zeros for the $L_{y}=3$ square strip in unpublished work.

[29] M. Roček, R. Shrock, and S.-H. Tsai, to appear.

[30] J. F. Nagle, J. Combin. Theory 10 (1971) 42; G. A. Baker, Jr., J. Combin. Theory 10 (1971) 217; D. Kim and I. G. Enting, J. Combin. Theory, B 26 (1979) 327. 
[31] B. Grünbaum and G. Shephard, Tilings and Patterns (Freeman, New York, 1987).

[32] V. Matveev and R. Shrock, J. Phys. A 28 (1995) 5235.

[33] V. Matveev and R. Shrock, Phys. Lett. A221 (1996) 343.

[34] S. Lefschetz, Algebraic Geometry (Princeton Univ. Press, Princeton, 1953); R. Hartshorne, Algebraic Geometry (Springer, New York, 1977).

[35] V. Matveev and R. Shrock, Phys. Lett. A204 (1995) 353.

[36] R. Shrock and S.-H. Tsai, Phys. Rev. E55 (1997) 5184.

[37] P. P. Martin, J. Phys. A 19 (1986) 3267; J. Phys. A 20 (1986) L601.

[38] E. J. Farrell, Discrete Math. 29 (1980) 161. 